\title{
25 Research Square \\ Targeting the USP1 dependent KDM4A protein stability as a potential prostate cancer therapy
}

\author{
Shu-Zhong Cui \\ Guangzhou Medical University \\ Ling-Ling Fan \\ University of Maryland Medical Center \\ You-Qiang Li \\ University of Maryland Medical Center \\ Xin-Yan Geng \\ University of Maryland Medical Center

\section{De-Xue Fu} \\ University of Maryland Medical Center \\ Hao-Wu Jiang \\ Washington University in Saint Louis School of Medicine \\ songhuixu ( $\sim$ songhuixu007@gmail.com ) \\ University of Maryland Medical Center
}

\section{Research}

Keywords: USP1, Prostate cancer, KDM4A, Deubiquitination, Tumorigenesis

Posted Date: November 12th, 2019

DOI: https://doi.org/10.21203/rs.2.17158/v1

License: (c) (i) This work is licensed under a Creative Commons Attribution 4.0 International License.

Read Full License

Version of Record: A version of this preprint was published at Cancer Science on March 30th, 2020. See the published version at https://doi.org/10.1111/cas.14375. 


\section{Abstract}

Background: Prostate cancer is the most commonly diagnosed malignancy and second leading cause of cancer death in American men. The histone demethylase KDM4A is reported to be overexpressed and plays a vital in multiple cancers through controlling gene expression by epigenetic regulation of $\mathrm{H} 3 \mathrm{~K} 9$ or H3K36 methylation marks. The biological role and mechanism of KDM4A in regulating AR activity in prostate cancer, however, remain to be established.

Methods: KDM4A expression in PTEN knockout mouse was determined using western blotting, Real-time PCR and immunohistochemical. Functional assays, such as cell survival, cell colony formation, anchorage-independent growth and a xenograft tumor model were used to determine the oncogenic role of KDM4A in prostate cancer cells. Association of KDM4A with USP1 and the deubiquitination assay were determined by co-immunoprecipitation, immunofluorescent staining and immunoblotting. USP1KDM4A axis promotes AR-dependent c-Myc expression was further investigated using western blotting, chromatin immunoprecipitation and Real-time PCR. The specific USP1 inhibitor ML323 was used for assessing the role of USP1 in prostate cancer cell survival. Co-expression of KDM4A with USP1 was determined by immunohistochemical staining in 146 prostate cancer tissue samples.

Results: Herein, we reported KDM4A expression was upregulation in PTEN knockout mouse prostate tissue. Depletion of KDM4A in prostate cancer cells inhibited their proliferation and survival in vivo and vitro. Further studies reveal that USP1 is a deubiquitinase that regulates KDM4A K48-linked deubiquitin and stability. Interestingly, we found c-Myc was a key downstream effector of USP1-KDM4A/AR axis in driving prostate cancer cell proliferation. Notably, upregulation of KDM4A expression with high USP1 expression were observed in $70 \%$ of prostate tumors and inhibition of USP1 promotes prostate cancer cells response to therapeutic agent_enzalutamide. Our studies propose USP1 may be an anti-cancer therapeutic target in prostate cancer.

Conclusions: Our findings demonstrate that USP1 deubiquitinates and stabilizes KDM4A, which promotes recruitment of AR to the $c-M y c$ gene enhancer and highlight USP1 potential as an anti-cancer therapeutic target in prostate cancer. Keywords: USP1; Prostate cancer; KDM4A; Deubiquitination; Tumorigenesis

\section{Background}

Prostate cancer (PC) is a leading cause of death in American men, behind only lung cancer. Androgen deprivation therapy (ADT) is the standard-of-care treatment for advanced disease when surgical approaches or radiation fail [1]. Androgen receptor (AR), a member of the steroid hormone receptor family of ligand-activated nuclear transcription factors, plays a key role in prostate cancer initiation, progression and resistance to androgen-deprivation therapy [2-4]. AR drives prostate cancer growth by binding to androgen-responsive elements (AREs) of target genes and recruiting either co-activators or corepressors [5]. It has yet to be determined which and how AR co-activators regulate AR activity. 
KDM4A (also known as JMJD2A or JHDM3A) reportedly serves as an AR co-activator via specific histone $\mathrm{H} 3$ on lysine 9 (H3K9) or lysine 36 (H3K36) demethylation at promoters or enhancers of some AR target genes [6-8]. KDM4A also functions in promoting tumorigenesis via impairing DNA damage repair and inducing genomic instability [9]. These findings suggest KDM4A plays a vital role in development and progression of prostate cancer. Recent report KDM4A is overexpression in several types of cancer cells such as breast tumors [10] and lung cancer [11]. However, the regulatory mechanism of KDM4A expression upregulation in cancers remains unknown. Here, we aimed to identify the posttranslational modification controlling KDM4A stabilization and the mechanism of KDM4A in regulating AR activity, which can be exploited for potential therapeutic interventions.

Deubiquitinating enzymes (DUBs) are proteases that reverse protein ubiquitination, a process which is significant for normal homeostasis. Several DUBs are deregulated in certain types of human cancer and increasingly being regarded as candidates for drug discovery [12]. USP1, One of the best-characterized human DUBs, is overexpressed in gastric cancer, melanoma, cervical, sarcoma and lung cancer [12]. The best-characterized function of USP1 is as a regulator of the Fanconi anemia (FA) pathway, which USP1mediated deubiquitination of FANCD2 and FANCI [13-16]. Besides these DNA repair-related functions, USP1 also contributes to prevent bHLH-mediated differentiation, and thus maintain stem-cell characteristics in osteosarcoma cells by deubiquitinating ID proteins [17]. However, the novel substrates and regulators of USP1 in cancers remain largely unclear. So, numerous works are required to characterize the substrates of USP1 and to better understand the mechanisms of USP1 in certain types of human cancer.

In the present study, we discover that USP1 regulates cancer cell proliferation and response to therapeutic drugs through the KDM4A/AR-c-Myc pathway. Mechanistically, USP1 deubiquitinated and stabilized KDM4A, which promoted recruitment of AR to the c-Myc gene enhancer. In addition, inhibition of USP1 decreased prostate cancer proliferation and promoted response to therapeutic agent_enzalutamide in a KDM4A-dependent manner. Notably, KDM4A is upregulation in most prostate tumors from patients, which is positively correlated with the high expression of USP1, suggesting KDM4A may function as a potential prognostic marker and USP1 may be an anti-cancer therapeutic target in prostate cancer.

\section{Methods}

\section{Cell lines and regents}

HEK293T cells and prostate cancer cell lines CWR22RV1 (RV1), LNCAP, VCAP, LN95, C4-2B, DU145 and PC3 were purchased from American Type Culture Collection (ATCC). All cell lines were tested and authenticated by karyotyping analysis on 2 Jun 2018. Expression plasmids containing pCMV-FlagKDM4A, pCMV-Flag-USP1 and pCMV-Myc-USP1 were constructed as previously described [18], and pCMV-Myc- USP1 C90S mutant (a deubiquitinating enzyme activity-disrupted mutant, in which a Cys to Ser point mutation was introduced) was generated using a QuikChange Site-Directed Mutagenesis Kit 
(Stratagene) and validated by DNA sequencing. The HA-tagged ubiquitin (HA-ub) plasmid and mutation constructs were kindly provided by Prof. Jianfei Qi from the University of Maryland Cancer Center.

KDM4A (C37E5) Rabbit mAb \#5328, USP1 (D37B4) Rabbit mAb \#8033, c-Myc (E5Q6W) Rabbit mAb \#18583, Androgen Receptor (D6F11) XP® Rabbit mAb \#5153, Di-Methyl-Histone H3 (Lys9) (D85B4) XP® Rabbit mAb \#4658 and NKX3.1 (D2Y1A) XP® Rabbit mAb \#83700 were purchased from Cell Signaling Technology. Anti-ub (sc-8017) antibodies were purchased from Santa Cruz. Antibodies against HA (H9658), FLAG (F1804), and $\beta$-actin (A1978) were purchased from Sigma. The USP1 inhibitor ML323 was from Selleck Chemicals. CHX was purchased from Apexbio Company.

\section{Cell survival assay}

PC cells were transfected with the indicated plasmids. $1 \times 10^{4}$ cells were plated in 96 -well culture plates and cultured in RPMI 1640 medium supplemented with 10\% FBS. 96-well plates were read in an Epoch2 microplate reader (BioTek Instruments). The cell survival ratio calculated via MTS (3-(4,5dimethylthiazol-2-yl)-5-(3-carboxymethoxyphenyl)-2-(4-sulfophenyl)- 2H-tetrazolium) assay (Promega).

\section{Soft agar colony-formation assays}

PC cells were transfected with the indicated plasmids and then $1 \times 10^{4}$ cells were plated in $0.25 \%(\mathrm{w} / \mathrm{v})$ agarose with a base layer of $0.5 \%(\mathrm{w} / \mathrm{v})$ agarose. Both layers contained complete 1640 medium. After 2 weeks, colonies were counted by using a light microscope with at $4 \mathrm{x}$ magnification with a numerical aperture 0.10 objective lens (ECLIPSE 80i; Nikon).

\section{Immunoprecipitation}

Cells were lysed in NETN buffer (50 mM Tris-HCl pH 8.0, $150 \mathrm{mM} \mathrm{NaCl,1 \%} \mathrm{NP-40,1} \mathrm{mM} \mathrm{EDTA,} \mathrm{add}$ Proteinase Inhibitor Cocktail (MedChemExpress) freshly before use. The resulting lysate were then precleaned by $25 \mathrm{~mL}$ protein $\mathrm{G}$ sepharose beads (GE Healthcare) at $4^{\circ} \mathrm{C}$. Immunoprecipitation mixtures, including protein lysates, blocked protein $\mathrm{G}$ sepharose and the indicated antibodies or IgG control derived from the same species as the indicated antibody, were incubated on a rotating wheel at $4 \mathrm{C}$ overnight. Sepharose beads with the bound immunoprecipitates were collected and subjected to four times washes with the cold NETN buffer and then analyzed by WB assay.

\section{Chromatin Immunoprecipitation}

PC cells were cultured in complete RPMI 1640 medium and infected with lentiviral constructs as indicated in figure legends. Cells were pretreated as described in the figure legends before being crosslinked with $1 \%$ formaldehyde and then sonicated. Soluble chromatin was immunoprecipitated as described 
previously [19]. Primer used for ChIP was c-Myc enhancer 5'-CCAGCGAATTATTCAGAA-3', 5'AATTACCATTGACTTCCTC-3'. ReChIP analysis was performed as described previously [19]. Briefly, firstround antibody was added to chromatin extracts and incubated overnight at $4^{\circ} \mathrm{C}$ followed by addition of $60 \mathrm{ml}$ salmon sperm/protein A agarose (Upstate Biotechnology) to recover immunocomplexes. The bound protein complexes were eluted by $10 \mathrm{mM}$ dithiothreitol (DTT) at room temperature for $30 \mathrm{~min}$, and the elution was then diluted ten times with reChIP buffer (1\% Triton X-100, 2 mM EDTA, $150 \mathrm{mM} \mathrm{NaCl}, 20$ $\mathrm{mM}$ Tris [pH 8.1]) and subsequently reimmunoprecipitated by addition of the second-round or control IgG antibodies overnight at $4 \mathrm{C}$. Recovery and preparation of DNA was performed and followed by PCR using the oligonucleotides described above.

\section{Protein identification by mass spectrometry}

Whole cell lysates from RV1 cells stably expressing Flag-KDM4A were prepared using IP-lysis buffer. Immunoprecipitation of Flag-KDM4A was performed as described in Figure legend. The precipitated proteins were eluted with $3 \times$ flag peptides. The eluted samples were subject to in-solution trypsin digestion, LC-MS analysis and database search by the proteomic service of Shenzhen university. Identities of the co-precipitated proteins was performed as described previously [20].

\section{In vivo and in vitro deubiquitylation of KDM4A.}

For in vivo KDM4A ubiquitylation assay, cells were transfected with the indicated plasmids, then treated with $20 \mu \mathrm{M}$ MG132 for 8 hours. Cells were collected and lysed in NETN buffer plus $0.1 \%$ SDS, $20 \mu \mathrm{M}$ MG132, and protease inhibitors. Lysates were incubated with anti-KDM4A antibody for 3 hours and protein $A / G$ agarose beads for a further 8 hours at $4^{\circ} \mathrm{C}$. The precipitated proteins were then released from the beads by boiling for 10 minutes in SDS-PAGE loading buffer and were subjected to IB with the antiHA-ub antibody. For preparation of ubiquitinated KDM4A as the substrate for the in vitro deubiquitination assay, 293T cells were transfected with both Flag-KDM4A and HA-ubiquitin for 2 hours, and then were

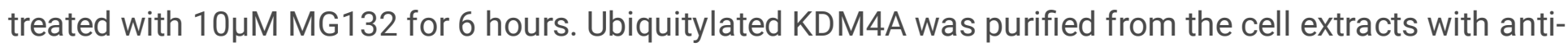
Flag breads and then incubated with the recombinant GST-USP1-WT or GST-USP1-C90S protein in a deubiquitylation buffer for 2 hours at $37^{\circ} \mathrm{C}$. Reactions were subjected to IB analysis.

\section{GST-tagged protein purification and GST pull-down assays.}

GST-USP1-WT and GST-USP1-C90S in the bacterial expression plasmid pGEX-4T-1 were expressed in the Escherichia coli strain BL21 by induction with $0.4 \mathrm{mM}$ isopropyl $\beta$-d-1-thiogalactopyranoside at $16^{\circ} \mathrm{C}$ and purified with GST beads (Sigma-Aldrich) according to the manufacturer's protocol. For GST pulldown assay, bacterial-expressed GST or GST-USP1-WT bound to glutathione-Sepharose 4B beads (GE 
Healthcare) was incubated with Flag-KDM4A expressed in HEK293T cells for 2 hours at $4^{\circ} \mathrm{C}$. After reaction, complexes were washed at least 4 times with GST-binding buffer, eluted by boiling in SDS-PAGE loading buffer, and subjected to IB with the indicated antibodies.

\section{Immunohistochemistry (IHC)}

Normal prostate tissue samples and prostate tumor tissue samples were collected at Affiliated Cancer Hospital \& Institute of Guangzhou Medical University. Tissue sample collection was approved by the Internal Review and Ethics Boards of Guangzhou Medical University. Tissue microarray chips containing normal prostate tissue samples and prostate tumor tissue samples were obtained from Shanghai OUTDO Biotech Co., Ltd. (Shanghai, China). Immunohistochemical staining was conducted as described previously [21]. The immunostaining was blindly scored by two pathologists. The immunohistochemical score was calculated as described previously [19]. The $\chi^{2}$ _test and Pearson's correlation coefficient were used for statistical analysis of the correlation between USP1 and KDM4A expression.

\section{Animal models and Mouse Xenograft Assays}

All in vivo studies were performed in accordance with the chinese Animals (Scientific Procedures) and approved by the Animal Welfare and Ethical Review Board at Guangzhou Medical University. The wild type mice and mice with prostate-specific deletion of PTEN (stock number 006440, Jackson Laboratories, USA) were kindly provided by Prof. Jianfei Qi from the University of Maryland Cancer Center. All mice housed under pathogen-free conditions and after 3 months were sacrificed when the standard situations occurred.

Seven-week-old male BALB/c nude mice were from Charles River Laboratories in China (Beijing). A total of $1 \times 10^{6} \mathrm{RV} 1$ cells stably expressing plasmids were injected subcutaneously into male BALB/c nude mice as standard procedure. Dr. Song-Hui Xu injected the cells into the mice and Dr. Shu-Zhong Cui, who were completely blinded to the experimental groups. After three weeks, we measured the tumors and analyzed the data.

The mice used in these experiments were bred and maintained under defined conditions at the Animal Experiment Center of the College of Medicine (SPF grade), Guangzhou Medical University. The animal experiments were approved by the Laboratory Animal Ethics Committee of The Guangzhou Medical University and conformed to the legal mandates and national guidelines for the care and maintenance of laboratory animals.

\section{Statistical analysis}


Statistical analysis was done by GraphPad software, version 5. Data are presented as the means \pm standard error of the means (SEM) or standard Deviation (SD). Student $t$ test was applied to assess the statistical significance. $P$ value $<0.05$ were considered

significant.

\section{Results}

\section{KDM4A expression regulates prostate cancer progression in vivo}

To investigate the role of KDM4A in prostate cancer, we firstly successfully established PTEN knockout (KO) mouse (hereafter, named as PTEN mice) model of prostate cancer. In this model, cre-recombinase is under the control of a modified rat prostate-specific probasin promoter (PB-Cre4) [22] that drives deletion of the floxed Pten allele in the epithelial cells of the mouse prostate. Prostate cancer development was monitored in the PTEN mice at 12 weeks of age, the spontaneous formation of prostate cancer was observed by histopathological analysis (Fig. 1a). Ki-67, a marker of cell proliferation. Ki-67 staining was significantly increased in prostate from the PTEN mice (Fig. 1b). To our interest, we found KDM4A protein were significantly increased in the PTEN mice compared to wild-type controls (Fig. 1c and 1e) but KDM4A mRNA level had no change (Fig. S1A). Previously reported KDM4A interacts with AR and functions as a key AR coactivator through its histone demethylase activity [6]. Here, in PTEN mice we found a greater increase in transcript levels of some AR target genes (i.e., c-Myc, NKX3.1, SPINK3 and SBP, but not SLC45A3, FKBP5 probasin and TMPRSS2), pointing to the possibility that KDM4A regulates the expression of a specific subset of AR target genes (Fig. 1d). The increase in lesions observed in PTENdeficient mice is not likely to be due to probasin driven SV40 T-antigen expression, as the level of probasin transcripts protein were similar in both wild-type mice and PTEN mice tissues (Fig. 1d). In agreement, the dorsal prostates from both genotypes expressed similar transcript levels of the luminal marker CK8 (Fig. 1d), suggesting that the reduced expression of AR target genes was not due to a change in cellular differentiation. Since increased KDM4A expression drives prostate cancer progression, the precise molecular mechanism how KDM4A activate AR in prostate cancer cell growth remains unclear. Here, we found the selected AR target gene, c-Myc and NKX3.1 expression are markedly increased in prostate tissue from PTEN mice compared to wild-type controls (Fig. 1e and S1B). These results suggest for the first time that KDM4A plays an oncogene role in prostate cancer progression, and that may link its role to AR signal.

\section{KDM4A knockdown inhibits cell proliferation and colony formation in vitro and tumor growth in vivo}


Given the abnormal expression of KDM4A in PTEN mice, we then assessed whether KDM4A expression affect PC progression. We used shRNA to inhibit KDM4A expression in two AR-positive PC cells (RV1 and LNCAP) and one AR-negative PC3 cells. Separately, both constructs reduced mRNA levels (Fig. S2A) and expression of KDM4A protein (Fig. 2a-2c). Further evaluating the role of KDM4A in PC proliferation, we found that PC cell proliferation was obviously decreased when KDM4A was knocked down in three PC cells (Fig. 2d and S2B). Concordantly, KDM4A knockdown also decreased the number and size of cell colonies in RV1 (Fig. 2e) and LNCAP cells (Fig. S2C). Consistent with this result, as determined in RV1 cells by soft agar colony formation assays (Fig. 2f). To determine whether KDM4A regulates AR activity in human PC cells, the knockdown of KDM4A RV1 cells were cultured in medium supplemented with 5\% charcoal stripped (CS)-fetal bovine serum (FBS) (which has low androgen levels) for two days and then stimulated with the synthetic androgen R1881 (to restore androgens to physiological levels) for 24 hrs. We found that protein levels of KDM4A were not affected irrespective of the androgen conditions, but AR protein levels were a little increasing in both RV1 and LNCAP cells after R1881 treatment (Fig. 2g and S2D). KDM4A knockdown has no effect on AR protein levels irrespective of the androgen conditions (Fig. $2 \mathrm{~g}$ and S2D). Furthermore, qRT-PCR analysis showed that KDM4A knockdown in RV1 cells reduced mRNA levels of the selected AR target genes (PSA, c-Myc and NKX3.1), but not others, under either androgendeprived or normal conditions (Fig. 2h). To further determine role of KDM4A in prostate cancer, we knocked down KDM4A in prostate cancer cells and measured the cell proliferation by colony formation assay or soft agar assay. Comparing with control cells, KDM4A knockdown in the AR-positive RV1 or LNCAP cells decreased the cell proliferation in the presence or absence of androgen (Fig. 2i, 2j and S2E). Of note, the effect of KDM4A knockdown to reduce cell growth was more apparent under the androgen deprivation condition (Fig. 2i, 2j and S2E).

To examine the in vivo pro-tumour activity by KDM4A, control vector or two stably expressed shKDM4A cells were individually injected into right subcutaneous tissues of Balb/c nude mice. Compared with control cells, KDM4A-knockdown RV1 cells showed a significantly reduction in tumor size (Fig. 2k) and weight (Fig. 2l). We next performed immunohistochemistry staining for the proliferation marker Ki67 on xenograft tumor sections. KDM4A knockdown reduced the percentage of Ki67-positive cells (Fig. 2m). These results suggest that KDM4A knockdown inhibits proliferation in xenograft prostate tumors, indicating that KDM4A participates in PC tumorigenesis.

\section{USP1 binds and stabilizes KDM4A}

To investigate the mechanism by which the KDM4A acts in cancer progression, the proteins that interact with KDM4A were identified and USP1, a deubiquitinase, was one of the top scored proteins identified by mass spectrometry (MS) analysis (Fig. 3a). The interaction between KDM4A and USP1 was confirmed by coimmunoprecipitation experiments in 293T cells overexpressing KDM4A (Fig. 3b). To determine whether KDM4A and USP1 directly interact, we mixed the purified GST-USP1 with the purified Flag-KDM4A and tested the in vitro binding between the two proteins. GST- USP1, but not GST protein, was co-precipitated with the Flag-KDM4A, indicating a direct interaction between the two proteins (Fig. S3A). Furthermore, 
immunofluorescence staining revealed that endogenous KDM4A and USP1 were mainly colocalized in punctate structures spreading throughout in the nucleus (Fig. 3c).

Multiple reports showed WDR48 act as a strong activator of USP1 by enhancing the USP1-mediated deubiquitination $[23,24]$. To test whether WDR48 is required for the interaction between KDM4A and USP1, we performed coimmunoprecipitation experiments in RV1 cells using KDM4A or WDR48 antibody pulldown assays. As shown in Fig. 3d and 3e, the interaction between KDM4A and WDR48 was confirmed by reciprocal coimmunoprecipitation experiments. In addition, the association of endogenous KDM4A with USP1 was reduced by WDR48 knockdown treatment in RV1 cells (Fig. 3f). As well known, active transcription foci are distributed in the nucleus in a punctate pattern; therefore, USP1, WDR48, KDM4A and AR may form a complex to regulate transcription in prostate cancer cells.

The interaction of USP1 and KDM4A led us to test a potential role for the ubiquitination enzyme USP1 in the regulation of KDM4A turnover and function. USP1 knockdown in RV1 cells, LNCAP and PC3 cells significantly decreased KDM4A protein level (Fig. 3g), but did not affect KDM4A mRNA level (Fig. S3B). In addition, treating cells with the proteasome inhibitor MG132 could not significantly reduce KDM4A protein level in cells down-regulation of USP1 (Fig. 3h). Furthermore, overexpression of wild-type (WT) USP1 but not the catalytic inactive mutant (CS mutant) in RV1 cells could dramatically increase KDM4A protein level in the cells depletion of USP1 (Fig. 3i). We then hypothesized that USP1 may regulate KDM4A stability. As shown in Fig. 3j, KDM4A protein was more stable after overexpression of USP1. Taken together, these results suggest that USP1 directly regulates KDM4A stability.

\section{USP1 deubiquitinates KDM4A}

To further investigate whether USP1 functions as a deubiquitin ligase for KDM4A, we performed a deubiquitination assay by co-transfecting cells with wild type USP1 or the CS mutant in the presence of MG132. A significant decrease in polyubiquitylated KDM4A protein was observed in cells transfected with wild type USP1, whereas the expression of CS mutant was not able to significantly reduce KDM4A ubiquitination (Fig. 4a). Conversely, using two different shRNA of USP1 dramatically increased KDM4A ubiquitination (Fig. 4b). Notably, the USP1 inhibitor, ML323 [25], significantly increased KDM4A ubiquitination (Fig. 4C). To determine whether USP1 functions as a directly deubiquitinase for KDM4A, ubiquitinated KDM4A was incubated with purified GST fusion USP1 (WT) or USP1 (CS) in vitro. As shown in Fig. 4d, a significant decrease in polyubiquitylated KDM4A protein was observed in cells transfected with GST fusion USP1, whereas GST fusion USP1 (CS) was not able to reduce KDM4A ubiquitination. Furthermore, we found that USP1 overexpression decreased the polyubiquitination levels in the wild type, K6R, K11R, K27R, K29R, K33R, and K63R HA-ubiquitin mutants but not in the K48R mutant (Fig. 4e), suggesting USP1 promotes KDM4A K48-linked deubiquitin, but which E3 ubiquitin Ligase mediated KDM4A ubiquitin and degradation is still unclear. Taken together, these results suggest USP1 is a new deubiquitinase that regulates KDM4A K48-linked deubiquitin and stability. 


\section{c-Myc is a downstream effector of USP1-KDM4A axis in PC cells}

Because of the key role of c-Myc in prostate cancer and our above findings (Fig. 2h) that KDM4A regulates c-Myc transcription, we determined whether c-Myc is a downstream effector of KDM4A. We knocked down KDM4A in two AR-positive PC cells (RV1 and LNCAP) and two AR-negative cells (PC3 and DU145). KDM4A knockdown in two AR-positive PC cells (RV1 and LNCAP) significantly decreased mRNA levels of C-Myc, whereas in other two AR-negative PC cells (PC3 or DU145) had no effect (Fig. 5a). Furthermore, western blot analysis showed that down-regulation of c-Myc expression, but not AR, following KDM4A knockdown in two AR-positive (Fig. 5b) or in AR-negative PC cells (Fig. 4S), suggesting that KDM4A regulates $\mathrm{c}-M y c$ expression at transcriptional and posttranscriptional levels. We previously found that USP1 knockdown decreased levels of KDM4A in prostate cancer cells. To evaluate the possible involvement of USP1-KDM4A axis in modulating c-Myc expression. We test whether KDM4A was able to rescue the effect caused by USP1 depletion. As shown in Fig. 5c, USP1 knockdown dramatically reduced levels of KDM4A and C-Myc, whereas the reconstitution of KDM4A was able to efficiently rescue their expression. In addition, our qPCR analysis also showed that USP1 knockdown in AR-positive PC cells decreased c-Myc mRNA levels, whereas the reconstitution of KDM4A was able to efficiently rescue the effect (Fig. 5d). Taken together, these results indicate that USP1 promotes the expression of c-Myc by regulating KDM4A. We next asked how USP1-KDM4A axis increases AR-dependent c-Myc transcription. Since KDM4A is a co-activator of AR [6], and the c-Myc gene enhancer contains an ARE [26], we performed a chromatin immunoprecipitation assay using a KDM4A antibody to determine whether KDM4A associates with the ARE on the c-Myc enhancer. We found silencing of either USP1, KDM4A, and or AR reduced KDM4A binding to c-Myc the enhancer (Fig. 5e). To evaluate whether KDM4A regulates AR binding to the c-Myc enhancer, we performed an AR chromatin immunoprecipitation assay. Downregulation of either USP1, KDM4A, and or AR decreased binding of AR to the c-Myc enhancer (Fig. 5f). Consistent with the role of KDM4A as the H3K9me2 demethylase, down-regulation of either USP1, KDM4A, and or AR increased levels of the H3K9me2 mark at the c-Myc enhancers (Fig. $5 \mathrm{~g}$ ). To determine whether USP1-KDM4A axis dependent transcription of c-Myc requires the AR ligand, we maintained the prostate cancer cells (control, shUSP1, shUSP1+KDM4A) in the media supplemented with charcoalstripped fetal bovine serum that contains extremely low levels of androgen for 2 days, followed by treatment of cells with or without synthetic androgen R1881 for 1 day. We found that R1881 induced NKX3.1 (a positive control) transcript significant increasing in RV1 cells, but it failed to induce the c-Myc transcript, and USP1 knockdown reduced the c-Myc or NKX3.1 transcript similarly with or without R1881, whereas the reconstitution of KDM4A was able to efficiently rescue the effect (Fig. 5h). These results suggest that AR ligand is not required for USP1- KDM4A axis dependent transcription of c-Myc. To further confirm this result, we performed chromatin immunoprecipitation assay using AR antibody in RV1 cells (control, shUSP1, shUSP1+KDM4A). Even in the absence of R1881, we observed the binding of AR to the c-Myc gene enhancer in RV1 control cells (control), and such binding was not affected by the treatment of R1881 (Fig. 5i). Knockdown of USP1 equally reduced the binding between AR and c-Myc enhancer in the 
presence or absence of R1881, the reconstitution of KDM4A partially rescue the effect (Fig. 5i), suggesting that USP1 not only regulates the binding between AR and c-Myc enhancer by KDM4A, but also by other transcription factor. Conversely, low level of AR was associated with NKX3.1 enhancer in the absence of R1881, while after R1881 treatment, the binding of AR to NKX3.1 enhancer was highly enhanced (Fig. 5i). Taken together, our results reveal and USP1-KDM4A axis is required for the constitutive association between AR and c-Myc enhancer in the presence or absence of androgen. This result is consistent with the previous report that AR up-regulates the expression of c-Myc in a ligandindependent manner [5].

To further test the role of USP1 in KDM4A-dependent tumor growth in vivo, we used a xenograft prostate tumor model in which RV1 cells were injected into nude mice. Compared with control cells, USP1 knockdown cells showed a significant decrease in tumor size (Fig. 5j) and weight (Fig. 5k) while the reconstitution of KDM4A in USP1 knockdown cells could significantly rescue xenograft tumor formation (Fig. 5j and 5k). In addition, we performed immunohistochemistry staining for the proliferation marker Ki67 on xenograft tumor sections (Fig. 5l). USP1 knockdown reduced the percentage of Ki67-positive cells while the reconstitution of KDM4A in USP1 knockdown cells could significantly rescue the effect (Fig. 5l).

Overall, these findings revealed that c-Myc was a key downstream effector of USP1 -KDM4A axis to drive the growth and survival of PC cells.

\section{KDM4A expression positively correlates with USP1 expression in clinical prostate cancer samples}

Previous studies have reported that KDM4A upregulation has been observed in prostate cancer $[9,27,28]$. As KDM4A plays a key role in human cancer development, it is possible that in human cancers USP1 promotes the deubiquitination and stabilization of KDM4A. To determine the relevance of regulation of KDM4A/AR-c-Myc signaling by USP1 in patients, we performed immunohistochemical staining of KDM4A and USP1 on the prostate cancer tissue microarrays. Firstly, we detect the expression of USP1 and KDM4A in PC cell lines and cancer tissue samples. As shown in Fig. 6a, USP1 protein levels were positively correlated with KDM4A expression either in AR positive or negative prostate cancer cells. Furthermore, western blot analysis showed high USP1 protein levels correlated with increased KDM4A expression in most gastric cancer samples (Fig. 6b). To determine the relevance of KDM4A regulation by USP1 in patients, we performed immunohistochemical staining of KDM4A and USP1 in prostate cancer tissue microarrays (TMAs) (Fig. 6c). Notably, upregulation of KDM4A expression and high USP1 expression were observed in $70 \%$ (102 of 146) and 75\% (109 of 146) of prostate tumors, whereas only $23 \%$ ( 8 of 35 ) and $26 \%$ (9 of 35) of normal prostate tissues exhibited high KDM4A expression and upregulation of USP1 expression (Fig. $6 \mathrm{~d}$ ). To our excited, a significant positive correlation $(R=0.405$, $p<0.001$ ) between the KDM4A and USP1 protein levels was observed in these prostate tumors: $74.5 \%$ (76 of 102) of tumors with high KDM4A expression also displayed up-regulation of USP1 expression (Fig. 
$6 d$ ). However, it should be noted that 25.5\% (26 of 102) of all tumor specimens had high KDM4A expression but low USP1 expression (Fig. 6d). Taken together, these results reveal that upregulation of USP1 expression may contribute to KDM4A overexpression in a substantial fraction of human prostate tumors, whereas in other prostate tumors, KDM4A can be activated by other posttranslational regulation, for example, down-regulation of miR-10 was correlation with high expression levels of KMD4A in human prostate tumors [29].

\section{Targeting USP1 promotes prostate cancer cells response to therapeutic agent_enzalutamide}

Our above findings suggest that targeting USP1-KDM4A axis may antagonize the growth of prostate cancer cells. Although specific KDM4A inhibitors are not available, while a selective special USP1 inhibitor_ML323 has been developed [25]. We asked whether ML323 could be used to target the KDM4A stability. Firstly, we found ML323 treatment of RV1 or LNCAP cells led to the down-regulation of KDM4A protein levels in a dose-dependent manner (Fig. 7a) and the reduced KDM4A levels were accompanied by the reduced levels of the KDM4A downstream targets c-Myc (Fig. 7a). Moreover, qPCR data analysis showed that $5 \mu \mathrm{M}$ ML323 treatment of RV1 or LNCAP cells reduced c-Myc mRNA levels, but not others (Fig. 7b). These results indicated that USP1 inhibitor_ML323 can destabilize KDM4A protein. To test the efficacy of ML323 on prostate cancer cell growth, we treated RV1 and LNCAP cells with various dosage of ML323 and performed clonogenic assay. As shown in Fig. 7c and S7A, inhibition of USP1 led to decreasing the colony formation.

As KDM4A regulates AR activity as an AR co-activator [6] and the second new-generation AR-pathway inhibitor_enzalutamide, extend patient life by only a few month [30,31]. Thus, we tested whether inhibition of USP1 by its pharmacological inhibitor or specific shRNA affects cell response to enzalutamide. Firstly, the RV1 and LNCAP cells were treated with vehicle or 5 $4 \mathrm{M}$ USP1 inhibitor and then responses to enzalutamide were subsequently measured. Firstly, we observed that the USP1 inhibitor promoted KDM4A degradation (Fig. 7d). Second, ML323 sensitized cells to enzalutamide treatment, whereas overexpression of KDM4A in the cells were significantly resistant to enzalutamide treatment even in the presence of ML323 (Fig. 7e and S5B). Similarly, USP1 knockdown in RV1 cells using two specific shRNAs significantly decreased KDM4A protein levels (Fig. 7f) and increased cellular sensitivity to enzalutamide treatment, whereas overexpression of KDM4A in depleted cells with USP1 reversed hypersensitivity to enzalutamide treatment (Fig. $7 \mathrm{~g}$ and S5C). These results reveal that targeting USP1 promotes prostate cancer cells response to therapeutic agent_enzalutamide.

Collectively, we demonstrate that USP1 interacts with KDM4A and promotes its deubiquitination, ultimately leading to KDM4A stabilization and inhibition of USP1 promotes prostate cancer cells response to therapeutic agent_enzalutamide (Fig. 7h). 


\section{Discussion}

Our study uncovers KDM4A is required to support increased cellular proliferation and cell survival in PTEN prostate tissue. USP1 is a deubiquitinase that regulates KDM4A K48-linked deubiquitin and stability. In addition, we found c-Myc serves as a key downstream effector of USP1-KDM4A/AR axis in driving prostate cancer cell proliferation as well as survival. These suggest KDM4A may function as a potential prognostic marker and USP1 may be an anti-cancer therapeutic target in prostate cancer.

KDM4A is up-regulation in various cancers including lung, breast, and colon and prostate cancers and its expression is vital for cell survival [10,11,32-36]. Here, we found that KDM4A expression is overexpression in PTEN knockout mouse prostate tissue and human prostate tumors, supports KDM4A drives prostate cancer progression. Moreover, we showed KDM4A knockdown decreased cell proliferation in vitro and vivo. These provide supportive evidence for KDM4A as a potential therapeutic target in prostate cancer. KDM4A reportedly serves as an AR co-activator and regulates gene expression through $\mathrm{H} 3 \mathrm{~K} 9$ and H3K36 demethylation [6-8]. Consistent with previous findings that KDM4A is an AR coactivator and AR upregulates c-Myc mRNA independent of its ligand in prostate cancer cells, we found that KDM4A can recruit AR to the c-Myc enhancer and demethylate H3K9me2 at that locus, whereas androgen is not required for the KDM4A-dependent binding of AR to c-Myc enhancer.

Whether KDM4A/AR-c-Myc pathway can be regulated in prostate cancer remains an unanswered question. Identifying new upstream effector of this pathway may contribute to improved understanding of the molecular events involved in prostate cancer progression and clarify new potential therapeutic targets in prostate cancer. Herein, by performing tandem affinity purification, we identified KDM4A binding protein, USP1, functions as a deubiquitinase and stabilizes KDM4A, which in turn facilitates AR recruiting to the c-Myc enhancer. Thus, our study identified a new player in AR signaling pathway. Furthermore, we found that USP1 knockdown reduced tumorigenesis and promoted prostate cancer cells response to therapeutic agent_enzalutamide in a KDM4A dependent manner. The supportive evidence as follows: Firstly, USP1 knockdown decreased prostate cancer cell proliferation in vitro, tumorigenesis in vivo, and promotes response to therapeutic agent_enzalutamide. Second, inhibition of USP1 sensitized cancer cells to therapeutic agent_enzalutamide, while this effect was blunted by overexpression of KDM4A. Collectively, our study demonstrates USP1 as a new upstream effector of KDM4A/AR-c-Myc pathway in prostate cancer. To our interest, USP1 reportedly destroys K63-linked poly-ubiquitin chains on histones and then promotes to recruit 53BP1 at DNA damage sites, while another previous finding that KDM4A regulates DNA repair by controlling the recruitment of 53BP1 at DNA damage sites and RNF8dependent degradation of KDM4A regulates DNA repair by controlling the recruitment of 53BP1 at DNA damage sites [37, 38]. Here, we found USP1 promoted K48-linked deubiquitination of KDM4A and stability in PC cells. These suggest USP1 may promote K48-linked deubiquitination of KDM4A, which abrogate the E3 ubiquitin ligases RNF8 ubiquitin KDM4A following the DNA damage. Further study will be needed to understand how RNF8 and USP1 coordinate KDM4A expression, which may explain why the activity of KDM4A is tightly regulated under physiological conditions. In addition, in our study, USP1 directly binds and stabilizes KDM4A but does not affect its transcription, suggesting deubiquitination is 
the major regulation of KDM4A by USP1. We then propose that KDM4A is upregulated in prostate cancer at the posttranscriptional level. Upregulation of USP1 maybe a potential mechanism. In fact, we observed that high expression of KDM4A was positively correlated with high USP1 expression in most human prostate tumors (Fig. 6d). These evidences strongly support our hypothesis and clarify a physiological relationship between KDM4A and USP1 in prostate cancer.

Androgen receptor normally activated by androgens is a vital for PC cells [39]. Blockade of AR signal pathway was shown to be an effective prostate cancer therapeutic strategy [30,40]. Enzalutamide, one of the most effective AR-directed therapies, recent report it could only increase overall survival about 2.5 to 5 months and the survival benefits of enzalutamide were achieved in only about $50 \%$ of prostate cancer patients treated [30, 31]. In addition, many Patients eventually develop enzalutamide-resistance, which results in a shorter survival $[41,42]$. To our excited, several studies report the deubiquitining enzyme USP1 inhibitor_ML323 could specially inhibit the deubiquitining activity of USP1 $[25,43,44]$ and we further showed the USP1 inhibitor_ML323 inhibits USP1-KDM4A/AR-c-Myc pathway, therefore decreases prostate cancer cell survival. Notably, we found combination treatment of enzalutamide and USP1 inhibitor gave more improvement in prostate cancer cell response. In future, we will test whether inhibition of USP1 in enzalutamide-resistance cells could promote prostate cancer cell response to enzalutamide.

In summary, this study identifies USP1 as a critical deubiquitinase for stabilizing KDM4A, thereby promoting PC growth and tumor progression. Anti-cancer effects of USP1 inhibition could be partially reversed by overexpression of KDM4A in vitro and in vivo. Interestingly, USP1 was positively correlated to KDM4A protein expression in prostate human tumors. Combination treatment of enzalutamide and targeting KDM4A stabilization through pharmacological inhibition of USP1 by ML323 may thus open an avenue for therapeutic intervention in prostate cancer patients.

\section{Conclusions}

In summary, we reported that KDM4A expression was upregulation in PTEN knockout mouse prostate tissue and identified KDM4A binding protein, USP1, functions as a deubiquitinase and stabilizes KDM4A, which in turn facilitates AR recruiting to the c-Myc enhancer. Our findings suggest inhibition of USP1 promotes prostate cancer cells response to therapeutic agent_enzalutamide and highlight USP1 potential as a target for prostate cancer therapy.

\section{Abbreviations}

KDM4A: Lysine-specific demethylase 4A; PC: Prostate cancer; NT: Normal mammary tissue; IHC: Immunohistochemistry; ADT: Androgen deprivation therapy; DUBs: Deubiquitinating enzymes; GST: Glutathione S transferase; AR: Androgen receptor; AREs: Androgen-responsive elements

\section{Declarations}




\section{Acknowledgements}

We thank Dr. Fuman Qiu and members of the Qiu Lab for helpful discussions. We thank Dr. Jianfei Qi for providing the information of the c-Myc enchancer sequence and HA-ub expression plasmids. We thank Dr. Fengbo Zhang for the Flag- KDM4A expression plasmids.

\section{Funding}

This work was supported by the Natural Science Foundation of Guangdong Province (No. 2017A030310413), National Natural Science Foundation of China (No. 81372493), Guangzhou Key Medical Discipline Construction Project Fund, Clinical Research Promotion Project of Guangzhou Medical University for Building High Level University, Educational Commission of Guangdong Province, China Project (No. 2015KTSCX114), and Guangzhou Science Technology and Innovation Commission (No. 2014Y2-00548 and 2014Y2-00152).

\section{Availability of data and materials}

All data generated or analysed during this study are included in this published article.

\section{Authors' contributions}

SHX conceived and designed the experiments, CSZ and SHX analyzed the data and prepared the manuscript; CSZ, LLF, XYG and DXF performed the experiments; HWJ and YQL provided the statistical support; CSZ and SHX collected the sample tissues and analyzed the IHC data. All authors read and approved the final manuscript.

\section{Ethics approval and consent to participate}

For the use of these clinical materials for research purposes, prior patient consent and approval from the clinical medical research center of the Guangzhou Medical University. Animal studies were approved by the Animal Welfare and Ethical Review Board at Guangzhou Medical University.

\section{Consent for publication}

Not applicable. 


\section{Competing interests}

The authors declare that they have no competing interests.

\section{References}

1.Harris WP, Mostaghel EA, Nelson PS, Montgomery B: Androgen deprivation therapy: progress in understanding mechanisms of resistance and optimizing androgen depletion. Nat Clin Pract Urol 2009, 6:76-85.

2.Mitsiades N: A road map to comprehensive androgen receptor axis targeting for castration-resistant prostate cancer. Cancer Res 2013, 73:4599-4605.

3.Sharma NL, Massie CE, Ramos-Montoya A, Zecchini V, Scott HE, Lamb AD, MacArthur S, Stark R, Warren AY, Mills IG, Neal DE: The androgen receptor induces a distinct transcriptional program in castrationresistant prostate cancer in man. Cancer Cell 2013, 23:35-47.

4.Cai C, He HH, Chen S, Coleman I, Wang H, Fang Z, Chen S, Nelson PS, Liu XS, Brown M, Balk SP: Androgen receptor gene expression in prostate cancer is directly suppressed by the androgen receptor through recruitment of lysine-specific demethylase 1. Cancer Cell 2011, 20:457-471.

5.Fan L, Peng G, Sahgal N, Fazli L, Gleave M, Zhang Y, Hussain A, Qi J: Regulation of c-Myc expression by the histone demethylase JMJD1A is essential for prostate cancer cell growth and survival. Oncogene $2016,35.2441-2452$.

6.Klose RJ, Kallin EM, Zhang Y: JmjC-domain-containing proteins and histone demethylation. Nat Rev Genet 2006, 7:715-727.

7.Murayama A, Ohmori K, Fujimura A, Minami H, Yasuzawa-Tanaka K, Kuroda T, Oie S, Daitoku H, Okuwaki M, Nagata K, et al: Epigenetic control of rDNA loci in response to intracellular energy status. Cell 2008, 133:627-639.

8.Shin S, Janknecht R: Activation of androgen receptor by histone demethylases JMJD2A and JMJD2D. Biochem Biophys Res Commun 2007, 359:742-746.

9.Berry WL, Janknecht R: KDM4/JMJD2 histone demethylases: epigenetic regulators in cancer cells. Cancer Res 2013, 73.2936-2942.

10.Berry WL, Shin S, Lightfoot SA, Janknecht R: Oncogenic features of the JMJD2A histone demethylase in breast cancer. Int J Oncol 2012, 41:1701-1706.

11. Mallette FA, Richard S: JMJD2A promotes cellular transformation by blocking cellular senescence through transcriptional repression of the tumor suppressor CHD5. Cell Rep 2012, 2:1233-1243. 
12.Garcia-Santisteban I, Peters GJ, Giovannetti E, Rodriguez JA: USP1 deubiquitinase: cellular functions, regulatory mechanisms and emerging potential as target in cancer therapy. Mol Cancer 2013, 12:91.

13.Nijman SM, Huang TT, Dirac AM, Brummelkamp TR, Kerkhoven RM, D'Andrea AD, Bernards R: The deubiquitinating enzyme USP1 regulates the Fanconi anemia pathway. Mol Cell 2005, 17:331-339.

14.Parmar K, Kim J, Sykes SM, Shimamura A, Stuckert P, Zhu K, Hamilton A, Deloach MK, Kutok JL, Akashi K, et al: Hematopoietic stem cell defects in mice with deficiency of Fancd2 or Usp1. Stem Cells 2010, 28:1186-1195.

15.Oestergaard VH, Langevin F, Kuiken HJ, Pace P, Niedzwiedz W, Simpson LJ, Ohzeki M, Takata M, Sale JE, Patel KJ: Deubiquitination of FANCD2 is required for DNA crosslink repair. Mol Cell 2007, 28:798-809.

16.Murai J, Yang K, Dejsuphong D, Hirota K, Takeda S, D'Andrea AD: The USP1/UAF1 complex promotes double-strand break repair through homologous recombination. Mol Cell Biol 2011, 31:2462-2469.

17.Williams SA, Maecker HL, French DM, Liu J, Gregg A, Silverstein LB, Cao TC, Carano RA, Dixit VM: USP1 deubiquitinates ID proteins to preserve a mesenchymal stem cell program in osteosarcoma. Cell 2011, 146:918-930.

18.Tang DE, Dai Y, Xu Y, Liu DZ, Hong XP, Ou ML, Jiang HW, Xu SH: The ubiquitinase ZFP91 promotes tumor cell survival and confers chemoresistance through FOXA1 destabilization. Carcinogenesis 2019.

19.Fan L, Zhang F, Xu S, Cui X, Hussain A, Fazli L, Gleave M, Dong X, Qi J: Histone demethylase JMJD1A promotes alternative splicing of AR variant 7 (AR-V7) in prostate cancer cells. Proc Natl Acad Sci U S A 2018, 115:E4584-E4593.

20.Xu SH, Zhu S, Wang Y, Huang JZ, Chen M, Wu QX, He YT, Chen, Yan GR: ECD promotes gastric cancer metastasis by blocking E3 ligase ZFP91-mediated hnRNP F ubiquitination and degradation. Cell Death Dis 2018, 9:479.

21.Tang DE, Dai Y, Lin LW, Xu Y, Liu DZ, Hong XP, Jiang HW, Xu SH: STUB1 suppresseses tumorigenesis and chemoresistance through antagonizing YAP1 signaling. Cancer Sci 2019, 110.3145-3156.

22.Wu X, Wu J, Huang J, Powell WC, Zhang J, Matusik RJ, Sangiorgi FO, Maxson RE, Sucov HM, RoyBurman P: Generation of a prostate epithelial cell-specific Cre transgenic mouse model for tissue-specific gene ablation. Mech Dev 2001, 101:61-69.

23.Goncalves JM, Cordeiro MMR, Rivero ERC: The Role of the Complex USP1/WDR48 in Differentiation and Proliferation Processes in Cancer Stem Cells. Curr Stem Cell Res Ther 2017, 12:416-422.

24.Raimondi M, Cesselli D, Di Loreto C, La Marra F, Schneider C, Demarchi F: USP1 (ubiquitin specific peptidase 1) targets ULK1 and regulates its cellular compartmentalization and autophagy. Autophagy 2019, 15:613-630. 
25.Yu Z, Song H, Jia M, Zhang J, Wang W, Li Q, Zhang L, Zhao W: USP1-UAF1 deubiquitinase complex stabilizes TBK1 and enhances antiviral responses. J Exp Med 2017, 214:3553-3563.

26.Gao L, Schwartzman J, Gibbs A, Lisac R, Kleinschmidt R, Wilmot B, Bottomly D, Coleman I, Nelson P, McWeeney S, Alumkal J: Androgen receptor promotes ligand-independent prostate cancer progression through c-Myc upregulation. PLoS One 2013, 8:e63563.

27.Kim TD, Jin F, Shin S, Oh S, Lightfoot SA, Grande JP, Johnson AJ, van Deursen JM, Wren JD, Janknecht R: Histone demethylase JMJD2A drives prostate tumorigenesis through transcription factor ETV1. J Clin Invest 2016, 126:706-720.

28.Wang LY, Hung CL, Chen YR, Yang JC, Wang J, Campbell M, Izumiya Y, Chen HW, Wang WC, Ann DK, Kung HJ: KDM4A Coactivates E2F1 to Regulate the PDK-Dependent Metabolic Switch between Mitochondrial Oxidation and Glycolysis. Cell Rep 2016, 16.3016-3027.

29.Mu H, Xiang L, Li S, Rao D, Wang S, Yu K: MiR-10a functions as a tumor suppressor in prostate cancer via targeting KDM4A. J Cell Biochem 2019, 120:4987-4997.

30.Scher HI, Fizazi K, Saad F, Taplin ME, Sternberg CN, Miller K, de Wit R, Mulders P, Chi KN, Shore ND, et al: Increased survival with enzalutamide in prostate cancer after chemotherapy. N Engl J Med 2012, 367:1187-1197.

31.Dhingra R, Sharma T, Singh S, Sharma S, Tomar P, Malhotra M, Bhardwaj TR: Enzalutamide: a novel anti-androgen with prolonged survival rate in CRPC patients. Mini Rev Med Chem 2013, 13:1475-1486.

32.Chu $\mathrm{CH}$, Wang LY, Hsu KC, Chen CC, Cheng HH, Wang SM, Wu CM, Chen TJ, Li LT, Liu R, et al: KDM4B as a target for prostate cancer: structural analysis and selective inhibition by a novel inhibitor. J Med Chem 2014, 57:5975-5985.

33.Cloos PA, Christensen J, Agger K, Maiolica A, Rappsilber J, Antal T, Hansen KH, Helin K: The putative oncogene GASC1 demethylates tri- and dimethylated lysine 9 on histone H3. Nature 2006, 442:307-311.

34.Kim TD, Shin S, Berry WL, Oh S, Janknecht R: The JMJD2A demethylase regulates apoptosis and proliferation in colon cancer cells. J Cell Biochem 2012, 113:1368-1376.

35.Li BX, Luo CL, Li H, Yang P, Zhang MC, Xu HM, Xu HF, Shen YW, Xue AM, Zhao ZQ: Effects of siRNAmediated knockdown of jumonji domain containing $2 A$ on proliferation, migration and invasion of the human breast cancer cell line MCF-7. Exp Ther Med 2012, 4:755-761.

36.Li BX, Zhang MC, Luo CL, Yang P, Li H, Xu HM, Xu HF, Shen YW, Xue AM, Zhao ZQ: Effects of RNA interference-mediated gene silencing of JMJD2A on human breast cancer cell line MDA-MB-231 in vitro. J Exp Clin Cancer Res 2011, 30:90. 
37. Ha K, Ma C, Lin H, Tang L, Lian Z, Zhao F, Li JM, Zhen B, Pei H, Han S, et al: The anaphase promoting complex impacts repair choice by protecting ubiquitin signalling at DNA damage sites. Nat Commun 2017, 8:15751.

38.Mallette FA, Mattiroli F, Cui G, Young LC, Hendzel MJ, Mer G, Sixma TK, Richard S: RNF8- and RNF168dependent degradation of KDM4A/JMJD2A triggers 53BP1 recruitment to DNA damage sites. EMBO J 2012, 31:1865-1878.

39.Gandhi J, Afridi A, Vatsia S, Joshi G, Joshi G, Kaplan SA, Smith NL, Khan SA: The molecular biology of prostate cancer: current understanding and clinical implications. Prostate Cancer Prostatic Dis 2018, 21.22-36.

40.Tran C, Ouk S, Clegg NJ, Chen Y, Watson PA, Arora V, Wongvipat J, Smith-Jones PM, Yoo D, Kwon A, et al: Development of a second-generation antiandrogen for treatment of advanced prostate cancer. Science 2009, 324:787-790.

41.Katzenwadel A, Wolf P: Androgen deprivation of prostate cancer: Leading to a therapeutic dead end. Cancer Lett 2015, 367:12-17.

42.Schalken J, Fitzpatrick JM: Enzalutamide: targeting the androgen signalling pathway in metastatic castration-resistant prostate cancer. BJU Int 2016, 117.215-225.

43.Liang Q, Dexheimer TS, Zhang P, Rosenthal AS, Villamil MA, You C, Zhang Q, Chen J, Ott CA, Sun H, et al: A selective USP1-UAF1 inhibitor links deubiquitination to DNA damage responses. Nat Chem Bio/ 2014, 10.298-304.

44.Dexheimer TS, Rosenthal AS, Liang Q, Chen J, Villamil MA, Kerns EH, Simeonov A, Jadhav A, Zhuang Z, Maloney DJ: Discovery of ML323 as a Novel Inhibitor of the USP1/UAF1 Deubiquitinase Complex. In Probe Reports from the NIH Molecular Libraries Program. Bethesda (MD)2010

\section{Figures}


a

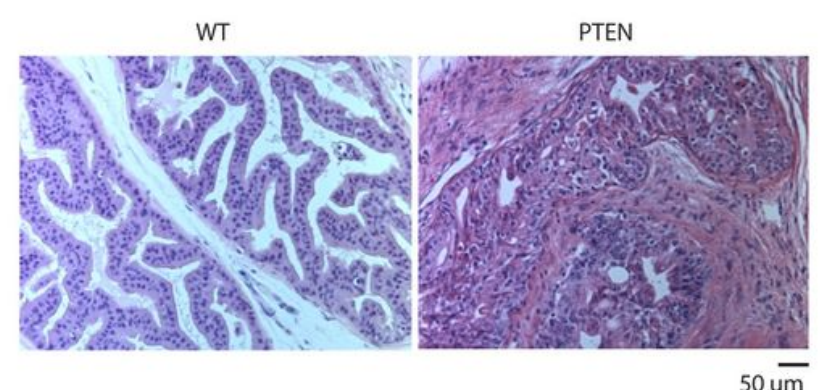

C

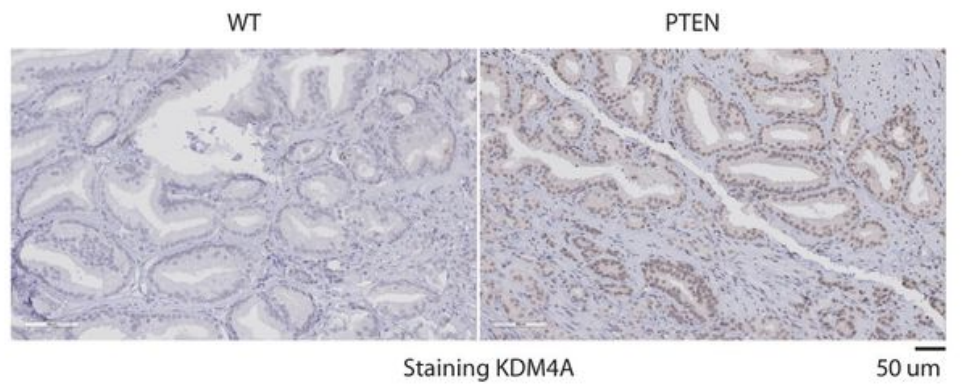

e

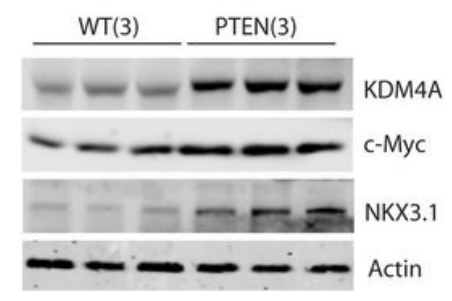

b
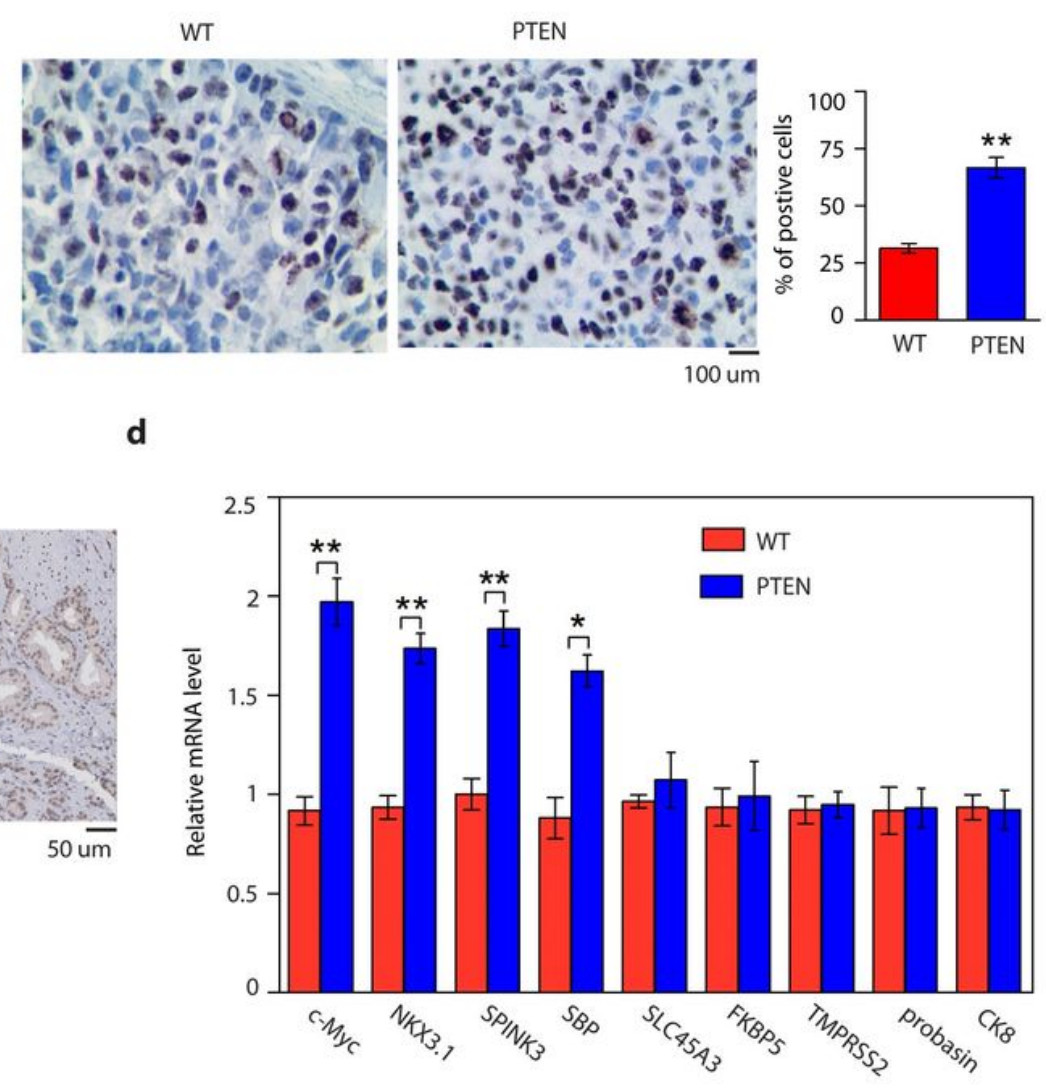

Figure 1

KDM4A expression regulates prostate cancer progression in vivo a. $\mathrm{H}+\mathrm{E}$ stained the dorsal prostates from 3 months aged wild-type (WT) and pbsn+ PTEN-/- male mice (PTEN). b. Percentage of Ki-67-positive cells in prostates from above. ${ }^{*} \mathrm{P}<0.01$ significant differences between genotypes. c. Representative IHC images of KDM4A expression in WT and PTEN mice prostate tissues. $d$. Transcript levels of selected AR target genes in the prostates of WT and PTEN mice, RNA for qRT-PCR was isolated from the dorsal prostates of 3-month-old mice. ${ }^{*} p<0.05$; ${ }^{*} p<0.01$. Data are mean \pm SD. e. Representative western blot showing the indicated protein expression in prostates from WT and PTEN mice ( $n=3$ mice per genotype). 
a

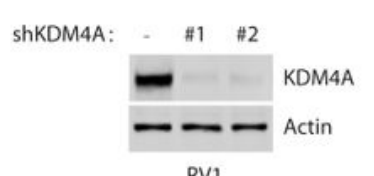

RV1 b

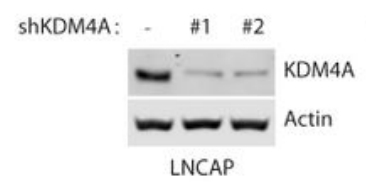

LNCAP c

ShKDM4A: - \#1 \#2

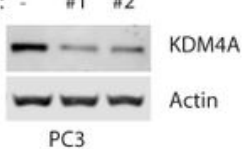

d

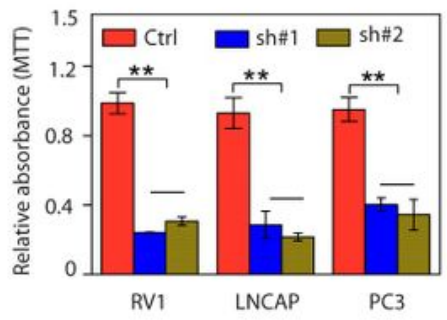

e

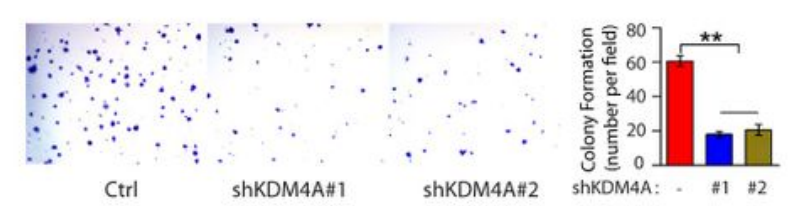

g

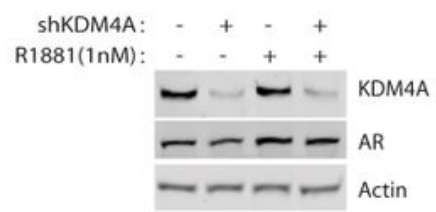

i

k

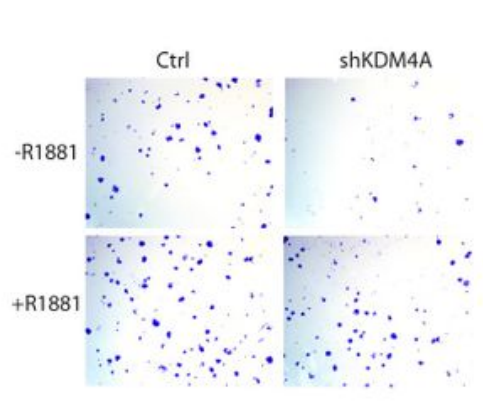

I
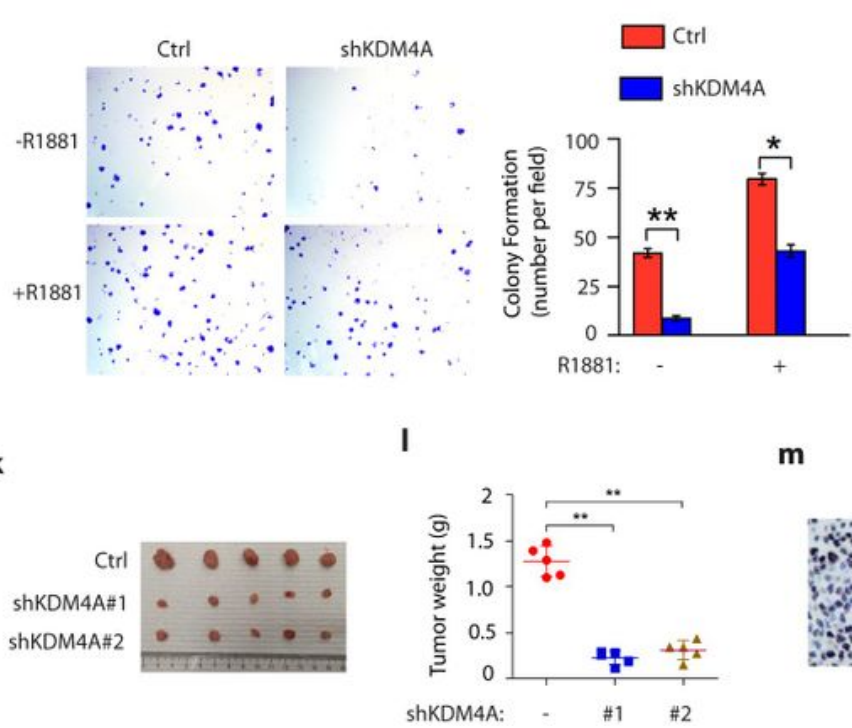

f
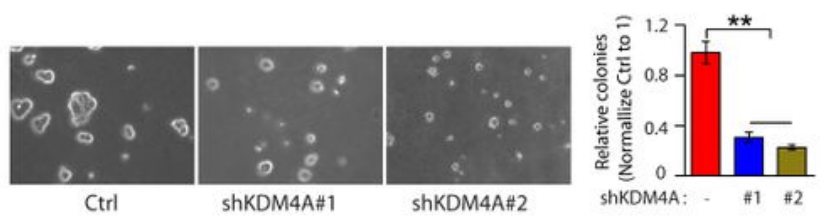

h

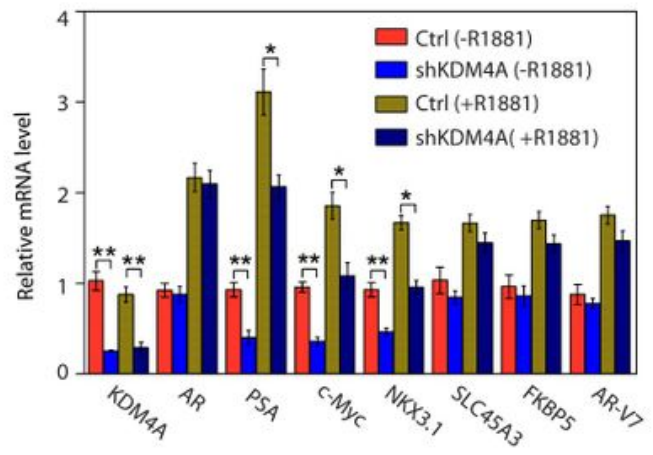

j

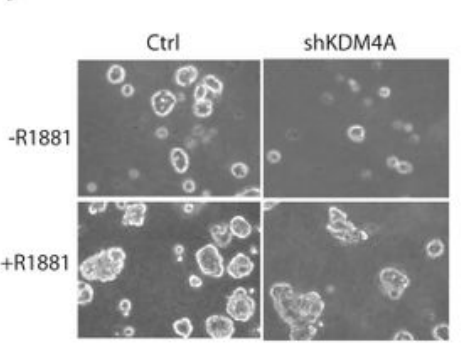

$\square$ PLKO.1

m
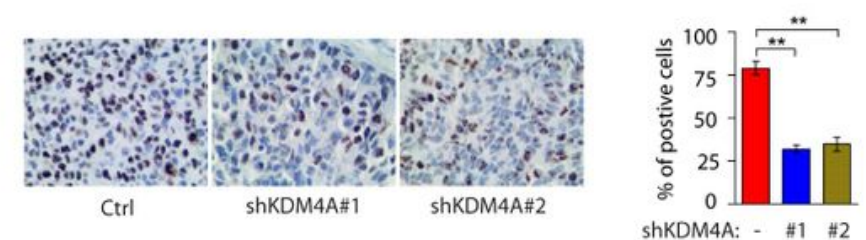

Figure 2

KDM4A knockdown inhibits cell proliferation and colony formation in vitro and tumor growth in vivo $a, b$, c. RV1 cells, LNCAP cells and PC3 cells stably expressing control (Ctrl) or KDM4A shRNAs (\#1 and \#2) were subjected to detect KDM4A expression. $d$. The viability of the above cells after stably expressing control (Ctrl) or KDM4A shRNAs (sh\#1 and sh\#2) was measured by MTT assay (mean \pm SEM of three independent experiments). ${ }^{* *} \mathrm{p}<0.01$. e. KDM4A knockdown decreased the colony formation by Rv1 cells 
on the cell-culture plate. Rv1 cells (Ctrl or KDM4A knockdown) were seeded at low density on the plate, and maintained for 2 weeks. The number of colonies was counted in 5 high-power fields (mean \pm SEM of three independent experiments). ${ }^{\star \star} p<0.01$. f. KDM4A knockdown decreased the colony formation by Rv 1 cells in soft agar. Rv1 cells (Ctrl or KDM4A knockdown) were grown in sofa agar for 3 weeks. The growth condition and colony number quantification are as described in $E$ (mean \pm SEM of three independent experiments). ${ }^{* \star} p<0.01$. g. Rv1 cells were transduced with control or KDM4A shRNA for 24 hours. Cells were then maintained in the growth media containing $5 \%$ charcoal stripped FBS (CS-FBS) with or without supplement of $1 \mathrm{nM}$ R1881 (a synthetic androgen). Proteins were collected and analyzed by the western blot. h. RNAs from g were collected and analyzed by the qRT-PCR for the selected AR target genes (mean \pm SEM of three independent experiments). ${ }^{*} p<0.05 ;{ }^{* *} p<0.01$. i. The indicated RV1 cells from $g$ were seeded at low density on the plate, and maintained for 2 weeks. The number of colonies was counted in 5 high-power fields (mean \pm SEM of three independent experiments). ${ }^{*} p<0.05 ; * \star p<0.01$. j. The cells from $g$ were grown in sofa agar for 3 weeks. The growth condition and colony number quantification are as described in g. k, I. RV1 cells stably expressing ctrl or shKDM4A RNAs were injected into the BALB/c nude mice, mice as described in Methods. Tumor images and weight of the tumors are shown in the $k$ and $I$ (mean \pm SD of 5 mice). All of the statistical analyses were performed with the ANOVA. ${ }^{*} p<0.05 ;{ }^{* *} p<0.01$. $\mathrm{m}$. Example staining of Ki67 on the tumor sections derived from above. The staining was developed by DAB (brown) and counterstained by hematoxylin (blue). The percentage of Ki67-positive cells on the staining as described in Methods. ${ }^{* *} p<0.01$ for Ctrl vs shKDM4A (ANOVA). 
a

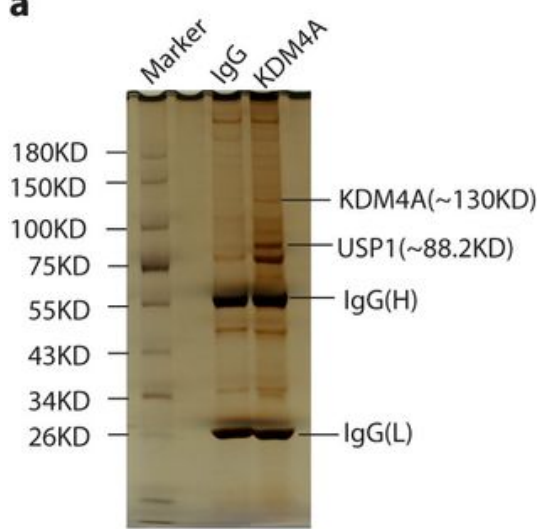

d

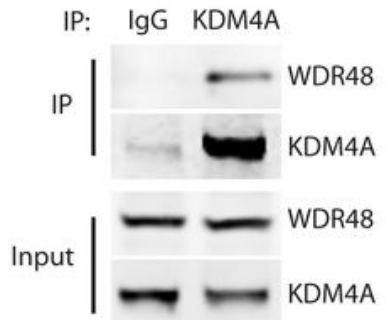

g

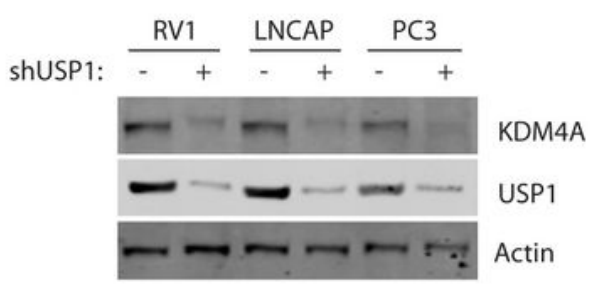

b

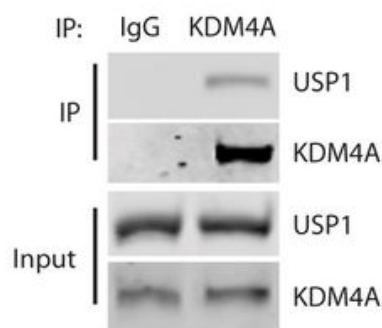

e

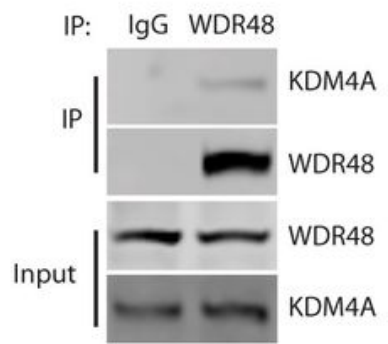

h

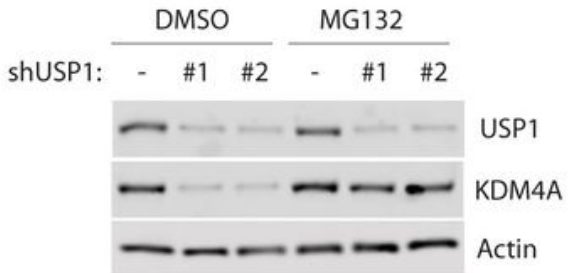

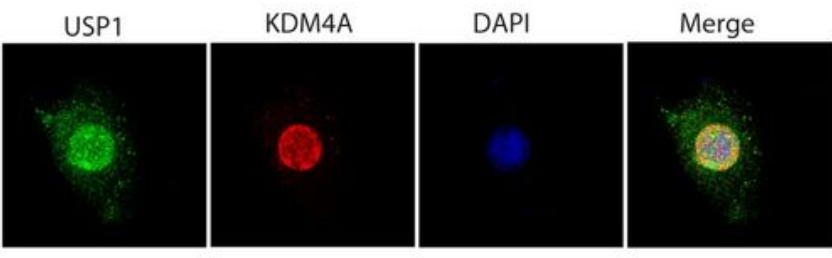

f
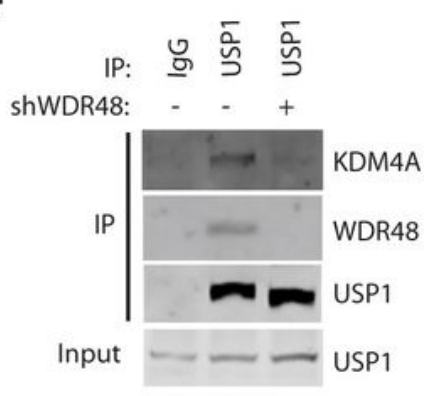

i Flag-USP1: - - WT Mut

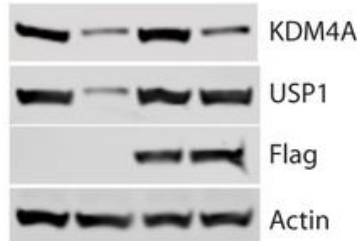

Figure 3

USP1 binds and stabilizes KDM4A a. Proteins that interacted with KDM4A were identified by Co-IP and mass spectrometry assays. b. RV1 cells were transfected with Flag-KDM4A plasmids for 48h, and lysates were subjected to immunoprecipitation with anti- KDM4A antobody. Bound proteins were analyzed by western blotting with anti-KDM4A or USP1 antibodies. c. Representative fluorescence images of KDM4A and USP1 proteins in RV1 cells. Cells were seeded on coverslips for 24h and then stained for KDM4A 
(red), and USP1 (green). Nuclei were visualized by DAPI staining (blue). d, e. RV1 cells were lysed and IPed with indicated antibodies. The immunocomplexes were subjected to western blotting. f. RV1 cells stably expressing control (ctrl) or WDR48 shRNAs were lysed and IPed with indicated antibodies. The immunocomplexes were subjected to western blotting. g. RV1 cells stably expressing control (Ctrl) or USP1 shRNAs were subjected to western blot to examine the indicated protein. $h$. The indicated cells were untreated or treated with $10 \mu \mathrm{M}$ MG-132 and western blotting was performed to examine the indicated protein levels. i. 293T cells were transfected with indicated constructs. The indicated proteins were analyzed by western blotting. j. RV1 cells were stably expressed control or Flag-USP1 constructs and then $\mathrm{CHX}$ pulse-chase assay was performed in cells. Right panel: the protein levels of USP1 relative to $\beta$-actin. 


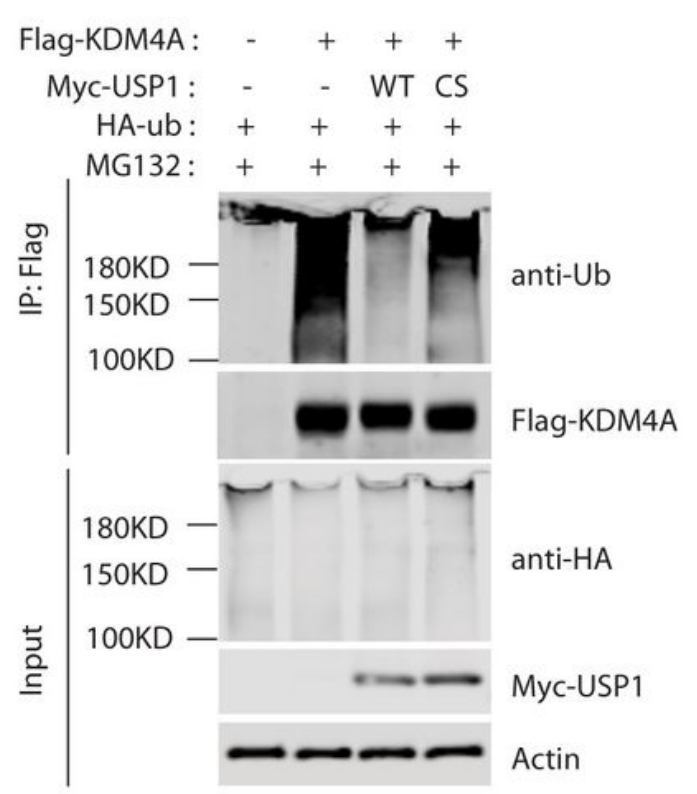

d

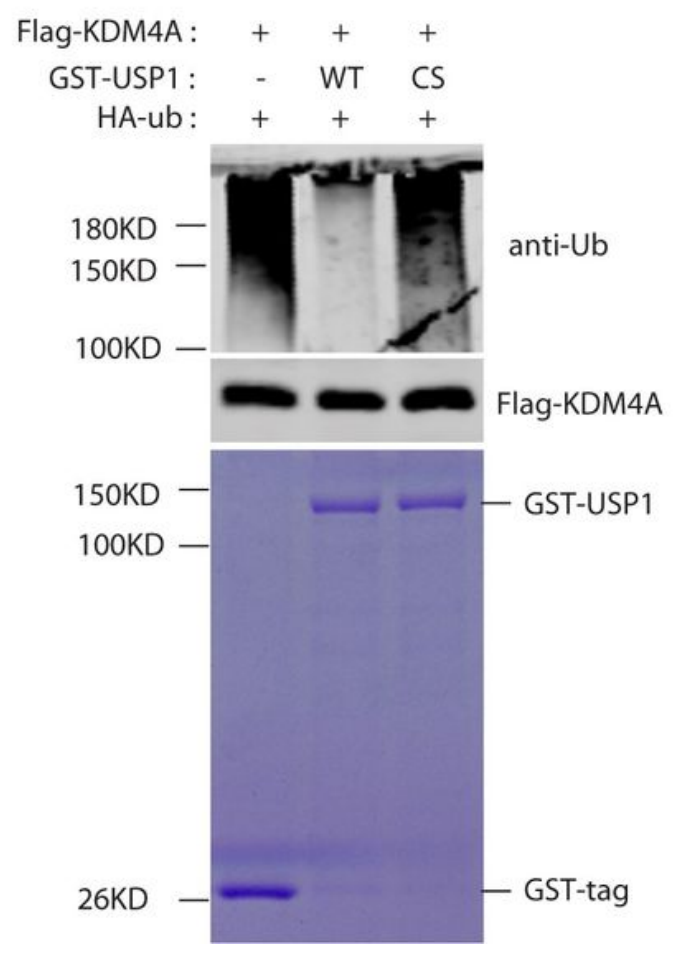

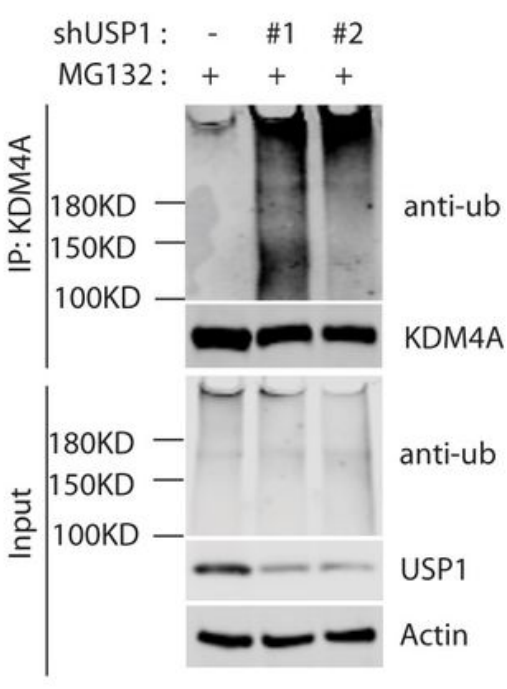

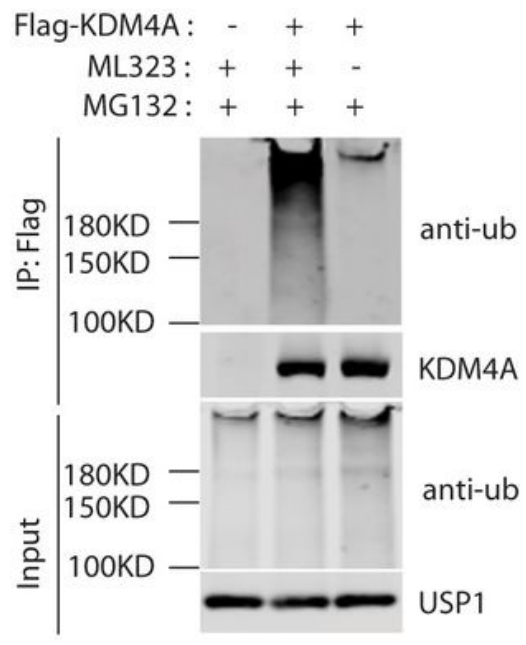

e

\section{Figure 4}

USP1 deubiquitinates KDM4A a. 293T cells were transfected with Flag-KDM4A, Myc-USP1 and HA-ub as indicated. Cellular lysates were co-immunoprecipitated by anti-Flag antibody. The polyubiquitylated KDM4A protein was detected by anti-HA-ub antibody. b. RV1 cells stably expressing control or USP1 shRNAs were subjected to ubiquitination assay and the polyubiquitylated KDM4A protein was detected by the anti-ub antibody. c. RV1 cells transfected with Flag-KDM4A were treated with or without $10 \mu \mathrm{M}$ 
ML323 (USP1 inhibitor). The polyubiquitylated KDM4A protein was examined as in b. d. Deubiquitination of KDM4A in vitro by USP1. Ubiquitinated KDM4A was incubated with purified GST fusion USP1 (WT) or USP39 (CS) in vitro and then blotted with indicated antibodies. e. 293T cells were transfected with MycUSP1, Flag-KDM4A and HA-ub (WT, K6R mutant, K11 mutant, K27 mutant, K29 mutant, K33 mutant, K48 mutant and $\mathrm{K} 63$ mutant). The analysis was performed as described $\mathrm{a}$.

a

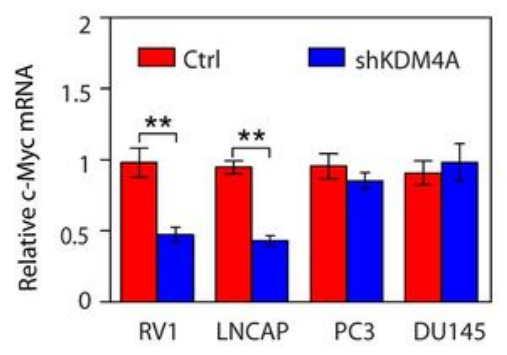

d

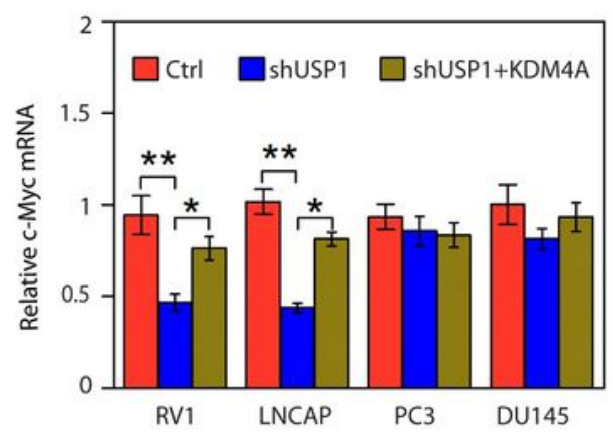

g

j

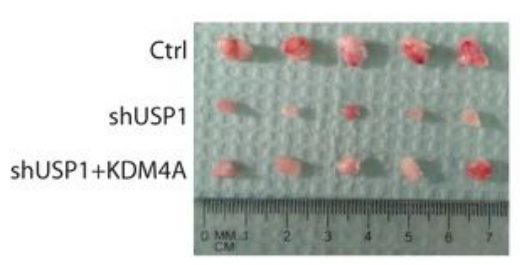

b

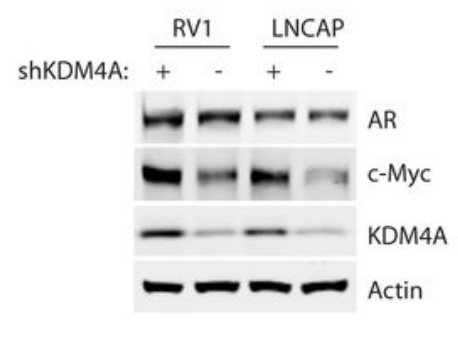

e

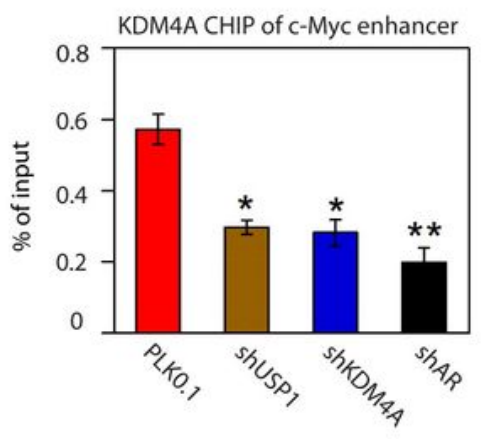

c

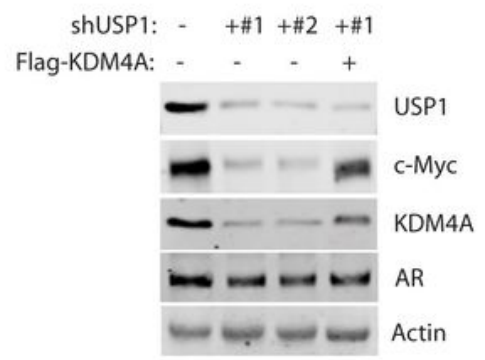

f

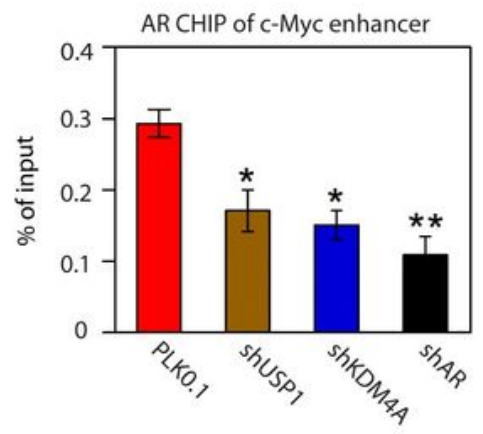

i

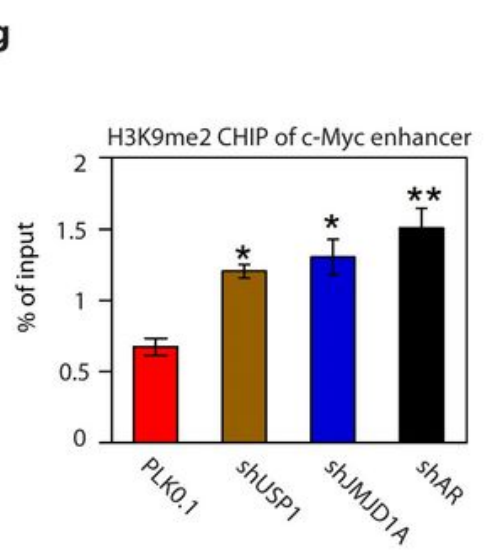

h

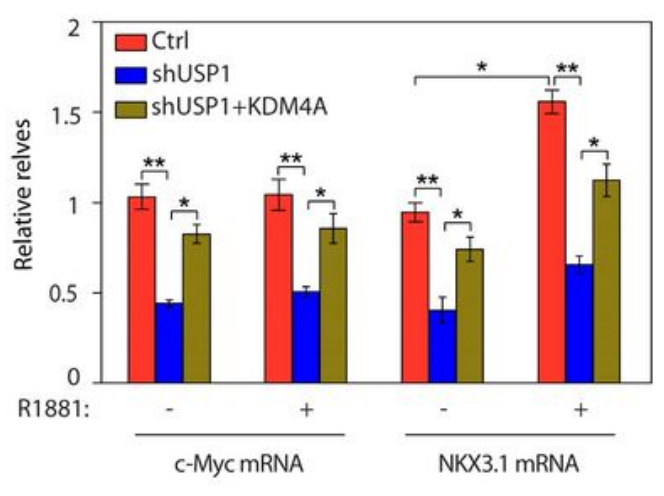

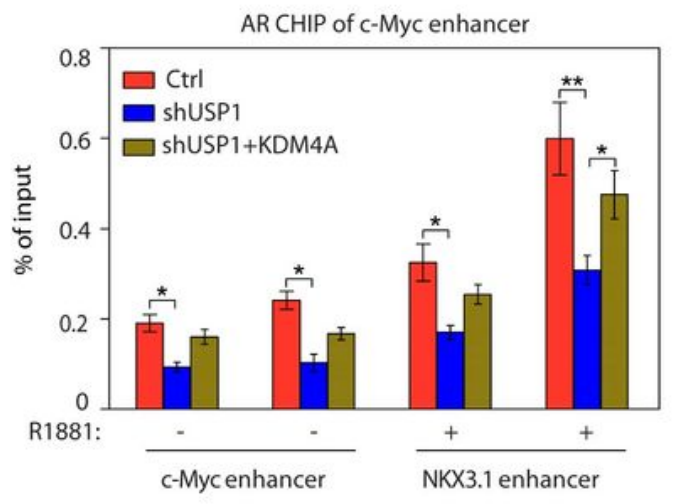

k

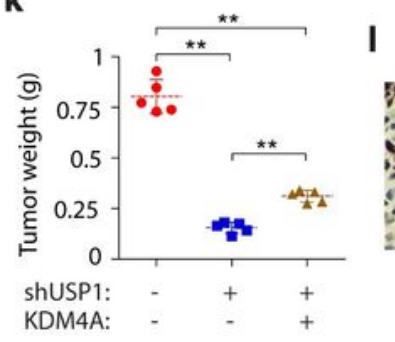

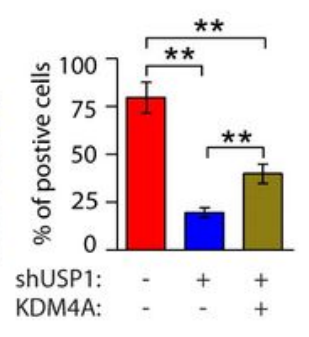

Figure 5 
c-Myc is a downstream effector of USP1-KDM4A axis in AR-positive cells a. AR-positive cells (RV1 cells, LNCAP cells) and AR-negative cells (PC3 cells, DU145) stably expressing control (Ctrl) or KDM4A shRNAs (\#1 and \#2) were subjected to detect mRNA level of c-Myc. ${ }^{* *} p<0.01$. b. The AR-positive cells (RV1 cells, LNCAP cells) described as A were subjected to western blot to examine the indicated protein. c. RV1 cells stably expressing ctrl or USP1 shRNAs together with or without Flag-KDM4A were analyzed by western blot. d. The indicated cells stably expressing ctrl or USP1 shRNAs together with or without Flag-KDM4A were subjected to detect mRNA level of $c-M y c$. ${ }^{*} p<0.05 ;{ }^{* *} p<0.01$. e. RV1 cells were transduced with USP1, KDM4A or AR shRNAs for $48 \mathrm{~h}$ and subjected to chromatin immunoprecipitation (ChIP) assay by antiKDM4A antibody. Chromatin was analyzed by qPCR for regions of the c-Myc enhancer exhibiting an ARE (androgen-responsive element). KD of either USP1, KDM4A or AR decreased KDM4A binding to ARE region. ${ }^{*} p<0.05 ;{ }^{*} p<0.01$. $f$. Cells described as E were subjected to chromatin immunoprecipitation (ChIP) assay by anti-AR antibody. Chromatin was analyzed by qPCR for regions of the c-Myc enhancer exhibiting an ARE. KD of either USP1, KDM4A or AR decreased AR binding to ARE region. * $p<0.05$; ${ }^{* *} p<0.01$. g. Cells described as $\mathrm{f}$. The chromatin immunoprecipitation (ChIP) assay by anti-H3K9Me2 antibody. KD of either USP1, KDM4A or AR decreased H3K9Me2 binding to ARE region. ${ }^{*} p<0.05 ;{ }^{* *} p<0.01$. h. RV1 cells stably expressing ctrl or USP1 shRNAs together with or without Flag-KDM4A were maintained in the growth media containing 5\% charcoal stripped FBS (CS-FBS) with or without supplement of $1 \mathrm{nM}$ R1881 for $48 \mathrm{~h}$ and then were subjected to detect mRNA level of c-Myc and NKX3.1. ${ }^{*}<<0.05 ;{ }^{*} p<0.01$. i. Cells described as $\mathrm{H}$ were subjected to chromatin immunoprecipitation (ChIP) assay by anti-AR antibody. Chromatin was analyzed by qPCR for regions of the c-Myc enhancer and NKX3.1 enhancer exhibiting AREs. j, k. RV1 cells stably expressing ctrl or shUSP1 RNAs were injected into the BALB/c nude mice, mice as described in Methods. Tumor images and weight of the tumors are shown in the $j$ and $k$ (mean \pm SD of 5 mice). All of the statistical analyses were performed with the ANOVA. ${ }^{*} p<0.05 ;{ }^{*} p<0.01$. I. Example staining of Ki67 on the tumor sections derived from above. The staining was developed by DAB (brown) and counterstained by hematoxylin (blue). The percentage of Ki67-positive cells on the staining as described in Methods. ${ }^{* *} p<0.01$ (ANOVA). 


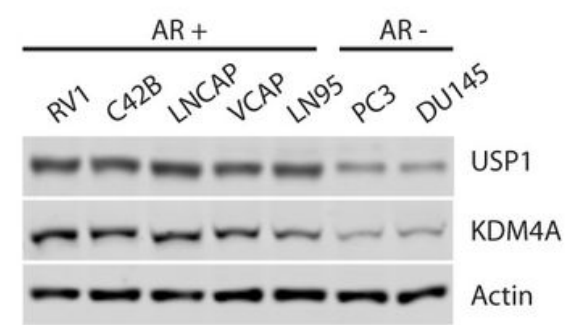

C

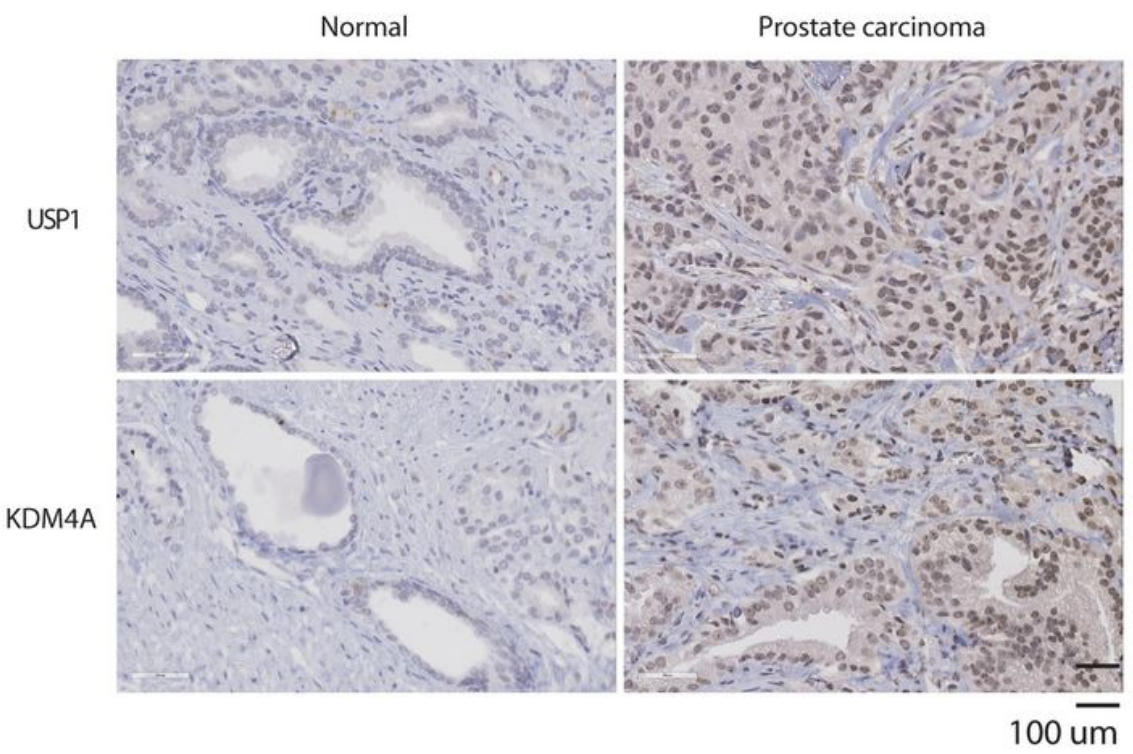

d

\begin{tabular}{lccc} 
& USP1 Low & USP1 High & Total \\
\hline Normal prostate & $26(74 \%)$ & $9(26 \%)$ & 35 \\
Prostate carcinoma & $37(25 \%)$ & $109(75 \%)$ & 146 \\
\hline $\mathrm{X}^{2}$ test, p<0.001 & & & \\
& KDM4A Low & KDM4A High & Total \\
\hline Normal prostate & $27(77 \%)$ & $8(23 \%)$ & 35 \\
Prostate carcinoma & $44(30 \%)$ & $102(70 \%)$ & 146 \\
\hline$X^{2}$ test, p<0.001 & & & \\
\hline KDM4A Low & 11 & 33 & 44 \\
KDM4A High & 26 & 76 & 102 \\
\hline$x^{2}$ test, p<0.001 & $\mathrm{R}=0.405$ & & USP1 High \\
\hline
\end{tabular}

$\mathrm{X}^{2}$ test, $\mathrm{p}<0.001, \mathrm{R}=0.405$

\section{Figure 6}

KDM4A expression positively correlates with USP1 expression in clinical prostate cancer samples a. Expression of USP1 and KDM4A in AR-positive cells (RV1 cells, C42B, LNCAP, VCAP and LN95 cells) and AR-negative cells (PC3 cells, DU145) was analyzed by western blot. b. A subset of the prostate tumor and normal tissues were subjected to western blotting, to examine the USP1 and KDM4A protein levels. C. Representative staining of USP1 and KDM4A in prostate cancer and normal prostate tissues. $\mathrm{d}$. Quantification of USP1 and KDM4A protein levels in normal and prostate cancer tissues, and the correlation study of USP1 and KDM4A expression level in prostate carcinoma. Statistical analyses were performed with the $\chi 2$-test, $p<0.001$. R: the Pearson's correlation coefficient. 


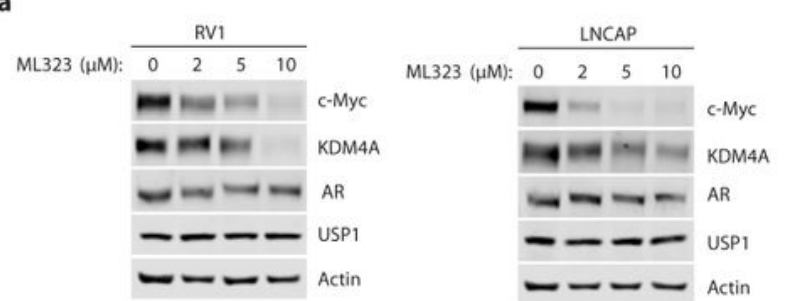

b

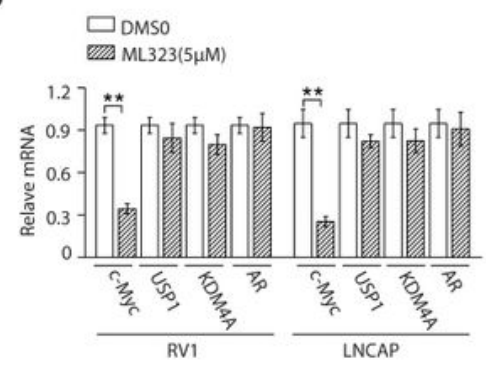

c
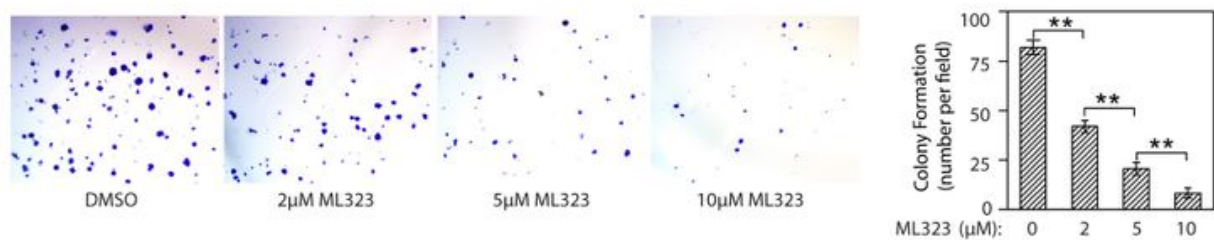

d

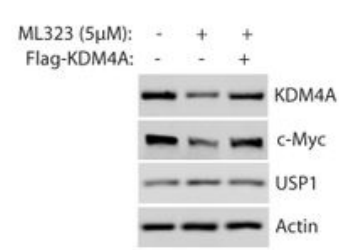

f

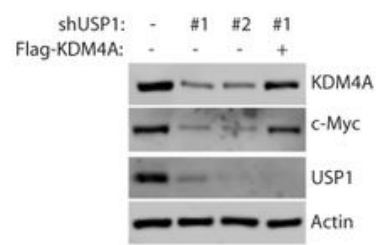

h
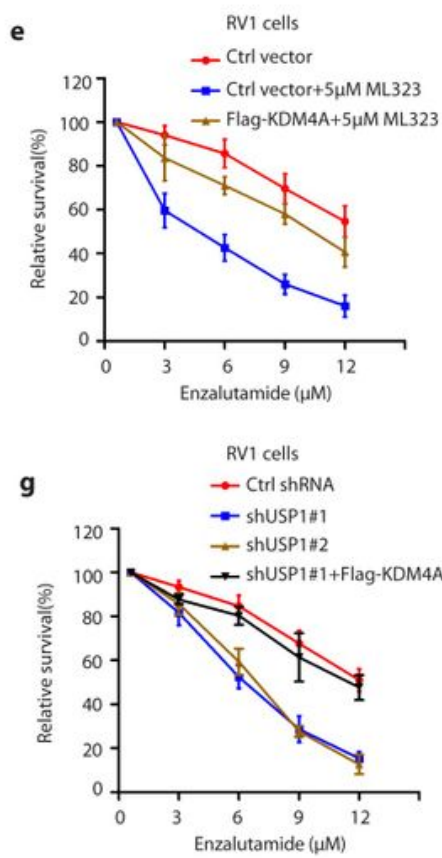
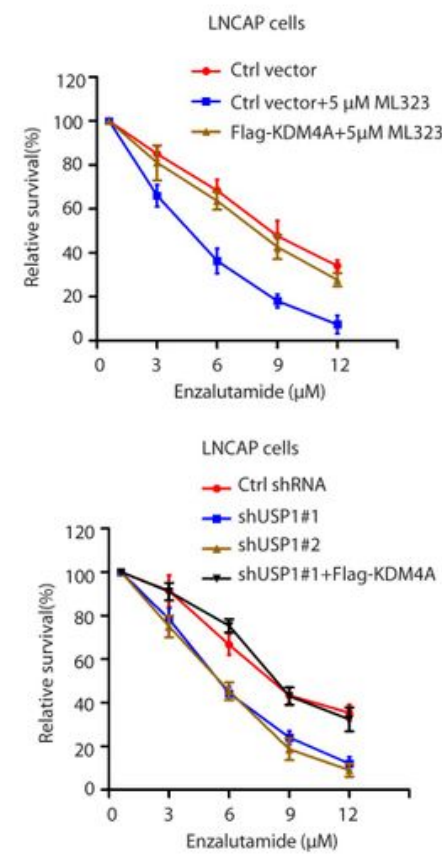

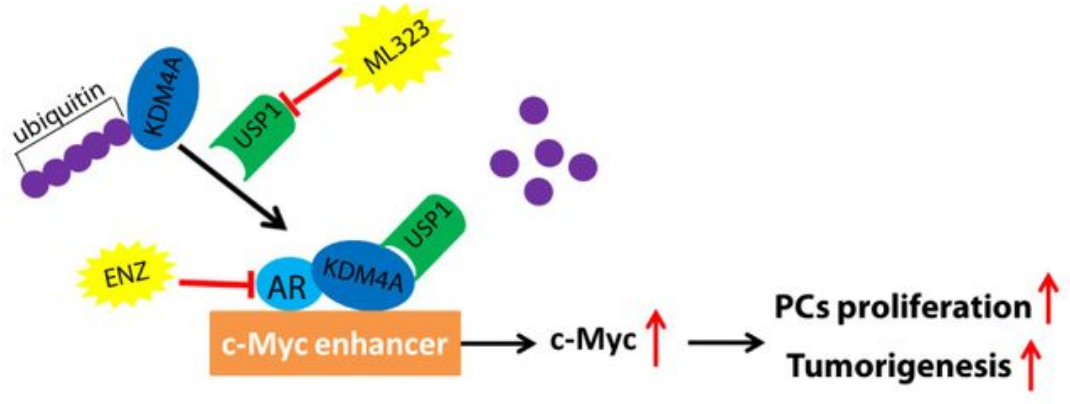

Figure 7

Targeting USP1 promotes prostate cancer cells response to therapeutic agent enzalutamide a. RV1 cells (left panel) or LNCAP (right panel) were treated with the indicated concentration of ML323 for 48 hours and analyzed by western blotting for the indicated proteins. b. Rv1 or LNCAP cells were treated with $5 \mu \mathrm{M}$ ML323 for 48 hours and were subjected to detect mRNA level of the indicated genes (mean \pm SEM of three independent experiments). ${ }^{*} p<0.05 ;{ }^{* \star} p<0.01$. c. RV1 cells described as a were seeded at low density 
on the plate, and maintained for 2 weeks. The number of colonies was counted in 5 high-power fields (mean \pm SEM of three independent experiments). ${ }^{\star \star} p<0.01$. d. RV1 cells were stably expressed with or without Flag-KDM4A constructs and treated with either vehicle or USP1 inhibitor ML323 for $24 \mathrm{~h}$. The cells were subjected to western blot to examine the indicated protein levels. e. Cells described as in $d$ were treated with the indicated concentration of enzalutamide and cell survival was determined (mean $\pm S D$ ( $n$ = 3)). f. RV1 cells stably expressing ctrl or USP1 shRNA together with or without Flag-KDM4A were subjected to western blotting to detect the indicated protein levels. $g$. Cells as in $f$ were treated with the indicated concentration of enzalutamide and cell survival was determined (mean $\pm S D(n=3)$ ). e. Schematic representation of how USP1 regulates AR and c-Myc activities via KDM4A.

\section{Supplementary Files}

This is a list of supplementary files associated with this preprint. Click to download.

- Supportinginformation.doc 